\title{
CRITICALITY AND SUBCRITICALITY OF GENERALIZED SCHRÖDINGER FORMS
}

\author{
MASAYOSHI TAKEDA
}

\begin{abstract}
Let $\mathcal{E}$ be a Dirichlet form and $\mu$ a positive Kato measure. We define criticality and subcriticality for the Schrödinger form, $\mathcal{E}(\cdot, \cdot)+(\cdot, \cdot)_{\mu}$, through $h$-transform. For a certain potential $\mu$, an analytic characterization of these properties is given in terms of the bottom of spectrum.
\end{abstract}

\section{Introduction}

Let $\mathcal{L}=-\nabla \cdot(A \nabla)+V$ be a Schrödinger operator on a domain $D$ of the $d$-dimensional Euclidean space $\mathbf{R}^{d}$ and $\mathcal{E}^{V}$ the associated Schrödinger form

$$
\mathcal{E}^{V}(u, u)=\int_{D}(A \nabla u, \nabla u) d x+\int_{D} u^{2} V d x, \quad u \in C_{0}^{\infty}(D) .
$$

Suppose that $\mathcal{E}^{V}$ is non-negative. Then Pinchover and Tintarev [11] prove the following dichotomy: $\mathcal{E}^{V}$ has a weighted spectral gap, that is, there exists a positive function $g>0$ such that $\int_{D} u^{2} g d x \leq \mathcal{E}^{V}(u, u)$, or there exist a sequence $\left\{\varphi_{n}\right\} \subset C_{0}^{\infty}(D)$ and a positive function $\varphi$ satisfying $\mathcal{L} \varphi=0$ such that $\varphi_{n}$ converges to $\varphi$ locally uniformly on $D$ and $\mathcal{E}^{V}\left(\varphi_{n}, \varphi_{n}\right)$ converges to zero. The former (resp. the latter) corresponds to that $\mathcal{L}$ is subcritical (resp. critical). Moreover, they establish an inequality of Poincaré-type in the latter case: there exist a positive function $g>0$ such that for any function $\psi \in C_{0}^{\infty}(D)$ with $\int_{D} \psi \varphi d x \neq 0$ there exists a constant $C>0$ such that

$$
\frac{1}{C} \int_{D} u^{2} g d m \leq \mathcal{E}^{V}(u, u)+C\left(\int_{D} u \psi d x\right)^{2} .
$$

Received March 28, 2014; received in final form May 12, 2014.

Partially supported by Grant-in-Aid for Scientific Research (No. 26247008(A)), Japan Society for the Promotion of Science.

2010 Mathematics Subject Classification. 31C25, 31C05, 60J25. 
One of our objectives is to extend these results to more general Dirichlet forms with non-local part. More precisely, let $X$ be a locally compact separable metric space and $m$ a positive Radon measure on $X$ with full topological support. Let $\mathbf{M}=\left(P_{x}, X_{t}\right)$ be an $m$-symmetric Hunt process. We assume that $\mathbf{M}$ is irreducible and strong Feller. We assume, in addition, that $\mathbf{M}$ generates a regular Dirichlet form $(\mathcal{E}, \mathcal{D}(\mathcal{E}))$ on $L^{2}(X ; m)$. Let $\mu=\mu^{+}-\mu^{-}$ be a suitable signed Radon measure such that the positive (resp. negative) part $\mu^{+}$(resp. $\mu^{-}$) belongs to the local Kato class (resp. the Kato class). We consider a Schrödinger form $\left(\mathcal{E}^{\mu}, \mathcal{D}\left(\mathcal{E}^{\mu}\right)\right)$ :

$$
\mathcal{E}^{\mu}(u, u)=\mathcal{E}(u, u)+\int_{X} u^{2} d \mu, \quad u \in \mathcal{D}\left(\mathcal{E}^{\mu}\right)\left(=\mathcal{D}(\mathcal{E}) \cap L^{2}\left(X ; \mu^{+}\right)\right) .
$$

In this paper, we define the criticality or subcriticality for $\mathcal{E}^{\mu}$ through $h$ transform; we denote by $A_{t}^{\mu}$ the positive continuous additive functional with Revez measure $\mu$, and define the Feynman-Kac semigroup $\left\{p_{t}^{\mu}\right\}_{t \geq 0}$ by

$$
p_{t}^{\mu} f(x)=E_{x}\left(e^{-A_{t}^{\mu}} f\left(X_{t}\right)\right) .
$$

We denote by $\mathcal{D}_{\text {loc }}(\mathcal{E})$ the set of functions locally in $\mathcal{D}(\mathcal{E})$ and introduce a function space by

$$
\mathcal{H}^{+}=\left\{h \in \mathcal{D}_{\text {loc }}(\mathcal{E}) \cap C(X): h>0, p_{t}^{\mu} h \leq h\right\} .
$$

Namely, $\mathcal{H}^{+}$is the space of $p_{t}^{\mu}$-excessive functions. Suppose that $\mathcal{H}^{+}$is not empty and take $h \in \mathcal{H}^{+}$. We then define the bilinear form $\left(\mathcal{E}^{\mu, h}, \mathcal{D}\left(\mathcal{E}^{\mu, h}\right)\right)$ on $L^{2}\left(X ; h^{2} m\right)$ through $h$-transform of $\left(\mathcal{E}^{\mu}, \mathcal{D}\left(\mathcal{E}^{\mu}\right)\right)$ :

$$
\left\{\begin{array}{l}
\mathcal{E}^{\mu, h}(u, u)=\mathcal{E}^{\mu}(h u, h u), \\
\mathcal{D}\left(\mathcal{E}^{\mu, h}\right)=\left\{u \in L^{2}\left(X ; h^{2} m\right): h u \in \mathcal{D}\left(\mathcal{E}^{\mu}\right)\right\} .
\end{array}\right.
$$

We see that $\left(\mathcal{E}^{\mu, h}, \mathcal{D}\left(\mathcal{E}^{\mu, h}\right)\right)$ turns out to be a regular Dirichlet form on $L^{2}\left(X ; h^{2} m\right)$. Consequently, if $\mathcal{H}^{+}$is not empty, then $\left(\mathcal{E}^{\mu}, \mathcal{D}\left(\mathcal{E}^{\mu}\right)\right)$ is nonnegative, $\mathcal{E}^{\mu}(u, u) \geq 0$ for all $u \in \mathcal{D}\left(\mathcal{E}^{\mu}\right)$. The semigroup $\left\{p_{t}^{\mu, h}\right\}_{t \geq 0}$ generated by $\left(\mathcal{E}^{\mu, h}, \mathcal{D}\left(\mathcal{E}^{\mu, h}\right)\right)$ is expressed as

$$
p_{t}^{\mu, h} f(x)=\frac{1}{h(x)} p_{t}^{\mu}(h f)(x) .
$$

The subcriticality, criticality and superciriticality for the Schrödinger form $\left(\mathcal{E}^{\mu}, \mathcal{D}\left(\mathcal{E}^{\mu}\right)\right)$ are defined as follows: $\left(\mathcal{E}^{\mu}, \mathcal{D}\left(\mathcal{E}^{\mu}\right)\right)$ is said to be subcritical (resp. critical) if $\mathcal{H}^{+}$is not empty and $\left(\mathcal{E}^{\mu, h}, \mathcal{D}\left(\mathcal{E}^{\mu, h}\right)\right)$ is transient (resp. recurrent) for some $h \in \mathcal{H}^{+}$. Besides, $\left(\mathcal{E}^{\mu}, \mathcal{D}\left(\mathcal{E}^{\mu}\right)\right)$ is said to be supercritical if $\mathcal{H}^{+}$is empty. We show that these definitions are well-defined (Lemma 4.2, Lemma 4.3). To this end, we prove the following Poincaré-type inequality: if $\left(\mathcal{E}^{\mu}, \mathcal{D}\left(\mathcal{E}^{\mu}\right)\right)$ is critical, then for any $h \in \mathcal{H}^{+}$there exist a nearly Borel finely closed set $B$ with $0<m(B)<\infty$ and a suitable strictly positive function $g$ 
such that for any $u \in \mathcal{D}\left(\mathcal{E}^{\mu}\right)$,

$$
\int_{X}\left(u-D_{h}(u) h\right)^{2} g d m \leq \mathcal{E}^{\mu}(u, u), \quad D_{h}(u)=\left\|h 1_{B}\right\|_{2}^{-2} \int_{B} u h d m
$$

(Theorem 3.2). As a result, the space $\mathcal{H}^{+}$is one-dimensional, for $h, \widetilde{h} \in \mathcal{H}^{+}$ there exists a positive constant $c$ such that $\widetilde{h}=c h$. The inequality (1.3) follows from [6, Theorem 4.8.2], which is an $L^{2}$-version of Oshima's inequality. If the Hunt process $\mathbf{M}$ admits a positive, jointly continuous transition density, we can derive from (1.3) an inequality similar to (1.1): for any bounded Borel function $\varphi$ with compact support and $\int_{X} \varphi d m \neq 0$ there exist a strictly positive continuous function $g$ in $L^{1}(X ; m)$ and a positive constant $C$ such that

$$
\frac{1}{C} \int_{X} u^{2} g d m \leq \mathcal{E}^{\mu}(u, u)+C\left(\int_{X} u \varphi d m\right)^{2}, \quad u \in \mathcal{D}\left(\mathcal{E}^{\mu}\right) .
$$

We further prove that if $\left(\mathcal{E}^{\mu}, \mathcal{D}\left(\mathcal{E}^{\mu}\right)\right)$ is subcritical, then there exists a positive function $g>0$ such that

$$
\int_{X} u^{2} g d m \leq \mathcal{E}^{\mu}(u, u), \quad u \in \mathcal{D}\left(\mathcal{E}^{\mu}\right)
$$

\section{(Theorem 3.7).}

The concept of extended Dirichlet spaces plays a crucial role in characterizing the recurrence and transience of Dirichlet forms. The criticality and subcriticality are generalized properties of the recurrence and transience respectively. Thus, we can easily imagine that for characterizing the criticality and subcriticality of Schrödinger forms, we need to introduce the concept of "extended Schrödinger spaces" of $\left(\mathcal{E}^{\mu}, \mathcal{D}\left(\mathcal{E}^{\mu}\right)\right)$. In fact, we define, through $h$-transform, the extended Schödinger form by (2.6) and study its properties (Lemma 2.8, Lemma 2.9). Furthermore, we can show that the inequalities (1.4) and (1.5) hold for all functions in the extended Schrödinger space. Owing to this extension, we can show that a Schrödinger form is critical if and only if there exists a positive function $h$ in the extended Schrödinger space and $\mathcal{E}^{\mu}(h, h)=0$. This fact is analogous to the fact that a Dirichlet form is recurrent if and only if the constant function 1 is in the extended Dirichlet space and $\mathcal{E}(1,1)=0$.

We denote by $\mathbf{M}^{\mu^{+}}=\left(P_{x}^{\mu^{+}}, X_{t}\right)$ the subprocess of $\mathbf{M}$ by the multiplicative functional $\exp \left(-A_{t}^{\mu^{+}}\right)$. We assume that if $(\mathcal{E}, \mathcal{D}(\mathcal{E}))$ is recurrent, then the positive part $\mu^{+}$is non-trivial, $\mu^{+} \not \equiv 0$. In other words, the subprocess $\mathbf{M}^{\mu^{+}}$is always assumed to be transient. In addition, the negative part $\mu^{-}$is supposed to be Green-tight with respect to $\mathbf{M}^{\mu^{+}}$(Definition 2.2(2)). Define $\lambda(\mu)$ by

$$
\lambda(\mu):=\inf \left\{\mathcal{E}(u, u)+\int_{X} u^{2} d \mu^{+}: u \in \mathcal{D}(\mathcal{E}), \int_{X} u^{2} d \mu^{-}=1\right\} .
$$


Then we can show that $\left(\mathcal{E}^{\mu}, \mathcal{D}\left(\mathcal{E}^{\mu}\right)\right)$ is subcritical, critical and supercritical, if and only if $\lambda(\mu)>1, \lambda(\mu)=1$ and $\lambda(\mu)<1$ respectively (Theorem 5.19). In fact, if $\lambda(\mu)>1$, then the gauge function $g^{\mu}(x)=E_{x}^{\mu^{+}}\left(\exp \left(A_{\zeta}^{\mu^{-}}\right)\right)$is bounded ([2, Theorem 5.1]). Besides, we can show that $g^{\mu}$ is an element of $\mathcal{H}^{+}$and that $\left(\mathcal{E}^{\mu, g^{\mu}}, \mathcal{D}\left(\mathcal{E}^{\mu, g^{\mu}}\right)\right)$ is transient (Lemma 5.5).

If $\lambda(\mu)=1$, the minimizer $h$ of (1.6) is a harmonic function, $\mathcal{E}^{\mu}(h, h)=0$. The existence of the minimizer follows from [14, Theorem 2.1]. We can show that the function $h$ belongs to $\mathcal{H}^{+}$and $\left(\mathcal{E}^{\mu, h}, \mathcal{D}\left(\mathcal{E}^{\mu, h}\right)\right)$ is recurrent; the function $h$ belongs to the extended Schrödinger space and $\mathcal{E}^{\mu}(h, h)=0$ if and only if the constant function 1 belongs to the extended Dirichlet space of $\left(\mathcal{E}^{\mu, h}, \mathcal{D}\left(\mathcal{E}^{\mu, h}\right)\right)$ and $\mathcal{E}^{\mu, h}(1,1)=0$.

For the proof that $h \in \mathcal{H}^{+}$we prove an inequality,

$$
\sup _{x \in X} G^{\mu} \nu(x) \leq \sup _{x \in K} G^{\mu} \nu(x) \cdot \sup _{x \in X} g^{\mu}(x),
$$

where $G^{\mu}(x, y)=\int_{0}^{\infty} p^{\mu}(t, x, y) d t, \nu$ is a smooth measure with topological support $K$. This inequality, which is of independent interest, is regarded as a version of the maximum principle; if $\mu^{-}=0$, that is, if $G^{\mu}$ is the Green kernel of the Markov process $P_{x}^{\mu^{+}}$, then $\sup _{x \in X} g(x)=1$ and (1.7) leads us to the equality,

$$
\sup _{x \in X} G^{\mu} \nu(x)=\sup _{x \in K} G^{\mu} \nu(x) .
$$

If the measure $\mu$ is negative, $\mu=-\mu^{-}$, then $\lambda(\mu)=1$ implies

$$
\int_{X} u^{2} d \mu \leq \mathcal{E}(u, u)
$$

and the equality in (1.8) holds for $h$. As a by-product of (1.3), we have a refinement of (1.8):

$$
\int_{X} u^{2} d \mu+\int_{X}\left(u-D_{h}(u) h\right)^{2} g d m \leq \mathcal{E}(u, u) .
$$

We see from $\left[15\right.$, Lemma 2.1] that $\left(\mathcal{E}^{\mu}, \mathcal{D}\left(\mathcal{E}^{\mu}\right)\right)$ is not non-negative definite if and only if $\lambda(\mu)<1$. Hence if $\lambda(\mu)<1$, then $\mathcal{H}^{+}$is empty and $\left(\mathcal{E}^{\mu}, \mathcal{D}\left(\mathcal{E}^{\mu}\right)\right)$ is supercritical.

We would like to comment on the space $\mathcal{H}^{+}$of superharmonic functions. We define the space of superharmonic functions in another way:

$$
\widetilde{\mathcal{H}}^{+}=\left\{h \in \mathcal{D}_{\text {loc }}(\mathcal{E}) \cap C(X): h>0, \mathcal{E}^{\mu}(h, \varphi) \geq 0, \forall \varphi \in \mathcal{D}(\mathcal{E}) \cap C_{0}^{+}(X)\right\} .
$$

This definition is more preferable than that of $\mathcal{H}^{+}$because it is directly related to $\left(\mathcal{E}^{\mu}, \mathcal{D}\left(\mathcal{E}^{\mu}\right)\right)$. If the Dirichlet form $(\mathcal{E}, \mathcal{D}(\mathcal{E}))$ is local, then the two spaces are identical, $\mathcal{H}^{+}=\widetilde{\mathcal{H}}^{+}$(Lemma 4.7). However, if $(\mathcal{E}, \mathcal{D}(\mathcal{E}))$ contains a jumping part, $\mathcal{E}(h, \varphi)$ is not well-defined for $h \in \mathcal{D}_{\text {loc }}(\mathcal{E})$ and $\varphi \in C_{0}(X)$. 


\section{Schrödinger forms}

Let $X$ be a locally compact separable metric space and $m$ a positive Radon measure on $X$ with full topological support. Let $(\mathcal{E}, \mathcal{D}(\mathcal{E}))$ be a regular irreducible Dirichlet form on $L^{2}(X ; m)$. We denote by $u \in \mathcal{D}_{\text {loc }}(\mathcal{E})$ if for any relatively compact open set $D$ there exists a function $v \in \mathcal{D}(\mathcal{E})$ such that $u=v m$-a.e. on $D$. We denote by $\mathcal{D}_{e}(\mathcal{E})$ the family of $m$-measurable functions $u$ on $X$ such that $|u|<\infty m$-a.e. and there exists an $\mathcal{E}$-Cauchy sequence $\left\{u_{n}\right\}$ of functions in $\mathcal{D}(\mathcal{E})$ such that $\lim _{n \rightarrow \infty} u_{n}=u m$-a.e. We call $\mathcal{D}_{e}(\mathcal{E})$ the extended Dirichlet space of $(\mathcal{E}, \mathcal{D}(\mathcal{E}))$.

Let $\mathbf{M}=\left(\Omega, \mathscr{F},\left\{\mathscr{F}_{t}\right\}_{t \geq 0},\left\{P_{x}\right\}_{x \in X},\left\{X_{t}\right\}_{t \geq 0}, \zeta\right)$ be the symmetric Hunt process generated by $(\mathcal{E}, \mathcal{D}(\mathcal{E}))$, where $\left\{\mathscr{F}_{t}\right\}_{t \geq 0}$ is the augmented filtration and $\zeta$ is the lifetime of M. Denote by $\left\{p_{t}\right\}_{t \geq 0}$ and $\left\{G_{\alpha}\right\}_{\alpha \geq 0}$ the semigroup and resolvent of $\mathbf{M}$ :

$$
p_{t} f(x)=E_{x}\left(f\left(X_{t}\right)\right), \quad G_{\alpha} f(x)=\int_{0}^{\infty} e^{-\alpha t} p_{t} f(x) d t .
$$

We assume that $\mathbf{M}$ satisfies next two conditions:

Irreducibility (I). If a Borel set $A$ is $p_{t}$-invariant, that is, $p_{t}\left(1_{A} f\right)(x)=$ $1_{A} p_{t} f(x) m$-a.e. for any $f \in L^{2}(X ; m) \cap \mathscr{B}_{b}(X)$ and $t>0$, then $A$ satisfies either $m(A)=0$ or $m(X \backslash A)=0$. Here $\mathscr{B}_{b}(X)$ is the space of bounded Borel functions on $X$.

Strong Feller Property $(\mathrm{SF})$. For each $t, p_{t}\left(\mathscr{B}_{b}(X)\right) \subset C_{b}(X)$, where $C_{b}(X)$ is the space of bounded continuous functions on $X$.

We remark that (SF) implies the following condition.

Absolute Continuity Condition (AC). The transition probability of $\mathbf{M}$ is absolutely continuous with respect to $m, p(t, x, d y)=p(t, x, y) m(d y)$ for each $t>0$ and $x \in X$.

Under (AC), there exists a non-negative, jointly measurable $\alpha$-resolvent kernel $G_{\alpha}(x, y)$ :

$$
G_{\alpha} f(x)=\int_{X} G_{\alpha}(x, y) f(y) m(d y), \quad x \in X, f \in \mathscr{B}_{b}(X) .
$$

Moreover, $G_{\alpha}(x, y)$ is $\alpha$-excessive in $x$ and in $y$ [6, Lemma 4.2.4]. We simply write $G(x, y)$ for $G_{0}(x, y)$. For a measure $\mu$, we define the $\alpha$-potential of $\mu$ by

$$
G_{\alpha} \mu(x)=\int_{X} G_{\alpha}(x, y) \mu(d y) .
$$

DeFInition 2.1.

(1) A Dirichlet space $(\mathcal{E}, \mathcal{D}(\mathcal{E}))$ on $L^{2}(X ; m)$ is said to be transient if there exists a strictly positive, bounded function $g \in L^{1}(X ; m)$ such that for 
$u \in \mathcal{D}(\mathcal{E})$

$$
\int_{X}|u| g d m \leq \sqrt{\mathcal{E}(u, u)}
$$

(2) A Dirichlet space $(\mathcal{E}, \mathcal{D}(\mathcal{E}))$ on $L^{2}(X ; m)$ is said to be recurrent if the constant function 1 belongs to $\mathcal{D}_{e}(\mathcal{E})$ and $\mathcal{E}(1,1)=0$. Namely, there exists a sequence $\left\{u_{n}\right\} \subset \mathcal{D}(\mathcal{E})$ such that $\lim _{n, m \rightarrow \infty} \mathcal{E}\left(u_{n}-u_{m}, u_{n}-u_{m}\right)=0$ and $\lim _{n \rightarrow \infty} u_{n}=1 m$-a.e.

For other characterizations of transience and recurrence, see [6, Theorem 1.6.2, Theorem 1.6.3].

We define the (1-)capacity Cap associated with the Dirichlet form $(\mathcal{E}, \mathcal{D}(\mathcal{E}))$ as follows: for an open set $O \subset X$,

$$
\operatorname{Cap}(O)=\inf \left\{\mathcal{E}_{1}(u, u): u \in \mathcal{D}(\mathcal{E}), u \geq 1, m \text {-a.e. on } O\right\}
$$

and for a Borel set $A \subset X$,

$$
\operatorname{Cap}(A)=\inf \{\operatorname{Cap}(O): O \text { is open, } O \supset A\},
$$

where $\mathcal{E}_{\alpha}(u, u)=\mathcal{E}(u, u)+\alpha(u, u)_{m}$. A statement depending on $x \in X$ is said to hold q.e. on $X$ if there exists a set $N \subset X$ of zero capacity such that the statement is true for every $x \in X \backslash N$. "q.e." is an abbreviation of "quasi-everywhere". A real valued function $u$ defined q.e. on $X$ is said to be quasi-continuous if for any $\epsilon>0$ there exists an open set $G \subset X$ such that $\operatorname{Cap}(G)<\epsilon$ and $\left.u\right|_{X \backslash G}$ is finite and continuous. Here, $\left.u\right|_{X \backslash G}$ denotes the restriction of $u$ to $X \backslash G$. Each function $u$ in $\mathcal{D}_{e}(\mathcal{E})$ admits a quasi-continuous version $\tilde{u}$, that is, $u=\tilde{u} m$-a.e. In the sequel, we always assume that every function $u \in \mathcal{D}_{e}(\mathcal{E})$ is represented by its quasi-continuous version.

Let $S_{00}$ be the set of positive Borel measures $\mu$ such that $\mu(X)<\infty$ and $G_{1} \mu$ is bounded. We call a Borel measure $\mu$ on $X$ smooth if there exists a sequence $\left\{E_{n}\right\}$ of Borel sets increasing to $X$ such that $1_{E_{n}} \cdot \mu \in S_{00}$ for each $n$ and

$$
P_{x}\left(\lim _{n \rightarrow \infty} \sigma_{X \backslash E_{n}} \geq \zeta\right)=1, \quad \forall x \in X,
$$

where $\sigma_{X \backslash E_{n}}$ is the first hitting time of $X \backslash E_{n}$. We denote by $S$ the set of smooth, positive Borel measures. In [6], a measure in $S$ is called a smooth measure in the strict sense. Here we omit the adjective phrase "in the strict sense".

A stochastic process $\left\{A_{t}\right\}_{t \geq 0}$ is said to be an additive functional (AF in abbreviation) if the following conditions hold:

(i) $A_{t}(\cdot)$ is $\mathscr{F}_{t}$-measurable for all $t \geq 0$.

(ii) There exists a set $\Lambda \in \mathscr{F}_{\infty}=\sigma\left(\bigcup_{t>0} \mathscr{F}_{t}\right)$ such that $P_{x}(\Lambda)=1$, for all $x \in X, \theta_{t} \Lambda \subset \Lambda$ for all $t>0$, and for each $\omega \in \Lambda, A$. $(\omega)$ is a function satisfying: $A_{0}=0, A_{t}(\omega)<\infty$ for $t<\zeta(\omega), A_{t}(\omega)=A_{\zeta}(\omega)$ for $t \geq \zeta$, and $A_{t+s}(\omega)=A_{t}(\omega)+A_{s}\left(\theta_{t} \omega\right)$ for $s, t \geq 0$. 
If an $\operatorname{AF}\left\{A_{t}\right\}_{t \geq 0}$ is positive and continuous with respect to $t$ for each $\omega \in \Lambda$, the $\mathrm{AF}$ is called a positive continuous additive functional (PCAF in abbreviation). The set of all PCAF's is denoted by $\mathbf{A}_{c}^{+}$. The family $S$ and $\mathbf{A}_{c}^{+}$ are in one-to-one correspondence (Revuz correspondence) as follows: for each smooth measure $\mu$, there exists a unique PCAF $\left\{A_{t}\right\}_{t \geq 0}$ such that for any $f \in \mathscr{B}^{+}(X)$ and $\gamma$-excessive function $h(\gamma \geq 0)$, that is, $e^{-\gamma t} p_{t} h \leq h$,

$$
\lim _{t \rightarrow 0} \frac{1}{t} E_{h \cdot m}\left(\int_{0}^{t} f\left(X_{s}\right) d A_{s}\right)=\int_{X} f(x) h(x) \mu(d x)
$$

[6, Theorem 5.1.7]. Here, $E_{h \cdot m}(\cdot)=\int_{X} E_{x}(\cdot) h(x) m(d x)$. We denote by $A_{t}^{\mu}$ the PCAF corresponding to $\mu \in S$. For a signed smooth measure $\mu=\mu^{+}-\mu^{-}$, we define $A_{t}^{\mu}=A_{t}^{\mu^{+}}-A_{t}^{\mu^{-}}$.

We define some classes of smooth measures.

Definition 2.2. Suppose that $\mu \in S$ is a positive Radon measure.

(1) $\mu$ is said to be in the Kato class of $\mathbf{M}(\mathcal{K}$ in abbreviation) if

$$
\lim _{\alpha \rightarrow \infty}\left\|G_{\alpha} \mu\right\|_{\infty}=0 \text {. }
$$

$\mu$ is said to be in the local Kato class $\left(\mathcal{K}_{\text {loc }}\right.$ in abbreviation) if for any compact set $K, 1_{K} \cdot \mu$ belongs to $\mathcal{K}$.

(2) Suppose that $\mathbf{M}$ is transient. A measure $\mu$ is said to be in the class $\mathcal{K}_{\infty}$ if for any $\epsilon>0$, there exists a compact set $K=K(\epsilon)$

$$
\sup _{x \in X} \int_{K^{c}} G(x, y) \mu(d y)<\epsilon .
$$

$\mu$ in $\mathcal{K}_{\infty}$ is called Green-tight.

We note that every measure treated in this paper is supposed to be Radon. Thus we see from $[1$, Theorem 3.9] that $\mu \in \mathcal{K}$ if and only if

$$
\lim _{t \downarrow 0} \sup _{x \in X} E_{x}\left(A_{t}^{\mu}\right)=\lim _{t \downarrow 0} \sup _{x \in X} \int_{0}^{t} \int_{X} p(s, x, y) \mu(d y) d s=0 .
$$

We denote the Green-tight class by $\mathcal{K}_{\infty}(G)$ if we would like to emphasize the dependence of the Green kernel. Chen [2] defines the Green-tight class in slightly different way; however the two definitions are equivalent under (SF) [8, Lemma 4.1]. We see from [13] that for $\alpha \geq 0$ and $\mu \in \mathcal{K}$

$$
\int_{X} u^{2} d \mu \leq\left\|G_{\alpha} \mu\right\|_{\infty} \cdot \mathcal{E}_{\alpha}(u, u) \quad \text { for any } u \in \mathcal{D}(\mathcal{E}) \text {. }
$$

Let $\mu=\mu^{+}-\mu^{-} \in \mathcal{K}_{\text {loc }}-\mathcal{K}$. We define the Schrödinger form by

$$
\left\{\begin{array}{l}
\mathcal{E}^{\mu}(u, u)=\mathcal{E}(u, u)+\int_{X} u^{2} d \mu, \\
\mathcal{D}\left(\mathcal{E}^{\mu}\right)=\mathcal{D}(\mathcal{E}) \cap L^{2}\left(X ; \mu^{+}\right) .
\end{array}\right.
$$

We denote by $\mathcal{L}^{\mu}=\mathcal{L}-\mu$ the self-adjoint operator associated with the closed symmetric form $\left(\mathcal{E}^{\mu}, \mathcal{D}\left(\mathcal{E}^{\mu}\right)\right),\left(-\mathcal{L}^{\mu} u, v\right)_{m}=\mathcal{E}^{\mu}(u, v)$. 
Lemma 2.3. $\mathcal{D}\left(\mathcal{E}^{\mu}\right) \cap C_{0}(X)=\mathcal{D}(\mathcal{E}) \cap C_{0}(X)$.

Proof. Noting that $\mu^{+}$is a Radon measure, we have

$$
\mathcal{D}\left(\mathcal{E}^{\mu}\right) \cap C_{0}(X)=\mathcal{D}(\mathcal{E}) \cap L^{2}\left(X ; \mu^{+}\right) \cap C_{0}(X)=\mathcal{D}(\mathcal{E}) \cap C_{0}(X) .
$$

We will establish the criticality theory for Schrödinger forms through $h$ transform. Let us introduce a function space:

$$
\mathcal{H}^{+}=\left\{h \in \mathcal{D}_{\text {loc }}(\mathcal{E}) \cap C(X): h>0, p_{t}^{\mu} h \leq h\right\} .
$$

Assume that $\mathcal{H}^{+}$is not empty. Then for $h \in \mathcal{H}^{+}$define the bilinear form $\left(\mathcal{E}^{\mu, h}, \mathcal{D}\left(\mathcal{E}^{\mu, h}\right)\right)$ on $L^{2}\left(X ; h^{2} m\right)$ by

$$
\left\{\begin{array}{l}
\mathcal{E}^{\mu, h}(u, u)=\mathcal{E}^{\mu}(h u, h u), \\
\mathcal{D}\left(\mathcal{E}^{\mu, h}\right)=\left\{u \in L^{2}\left(X ; h^{2} m\right): h u \in \mathcal{D}\left(\mathcal{E}^{\mu}\right)\right\} .
\end{array}\right.
$$

The next lemma is proved in the manner of [6, Theorem 6.3.2].

Lemma 2.4. For $\varphi \in \mathcal{D}(\mathcal{E}) \cap C_{0}(X)$, the function $\varphi / h$ belongs to $\mathcal{D}(\mathcal{E}) \cap$ $C_{0}(X)$.

Proof. Let $K$ be the support of $\varphi$ and $D$ an open set containing $K$. Put $c=1 /\left(\inf _{x \in K} h(x)\right)$. Then, for $(x, y) \in D \times D$

$$
\begin{aligned}
\left|\frac{\varphi}{h}(x)\right| & \leq c|\varphi(x)|, \\
\left|\frac{\varphi}{h}(x)-\frac{\varphi}{h}(y)\right| & \leq 2 c|\varphi(x)-\varphi(y)|+c^{2}|h(x) \varphi(x)-h(y) \varphi(y)| .
\end{aligned}
$$

Since $\varphi$ and $h \varphi$ belong to $\mathcal{D}(\mathcal{E})$, the function $\varphi / h$ also belongs to $\mathcal{D}(\mathcal{E})$ by $[6$, Theorem 1.5.2(ii)].

Lemma 2.5. $\mathcal{D}\left(\mathcal{E}^{\mu, h}\right) \cap C_{0}(X)=\mathcal{D}(\mathcal{E}) \cap C_{0}(X)$.

Proof. By the definition of $\mathcal{D}\left(\mathcal{E}^{\mu, h}\right)$ and Lemma $2.3, u \in \mathcal{D}\left(\mathcal{E}^{\mu, h}\right) \cap C_{0}(X)$ if and only if $u h \in \mathcal{D}(\mathcal{E}) \cap C_{0}(X)$. Moreover, it follows from Lemma 2.4 that $u h \in \mathcal{D}(\mathcal{E}) \cap C_{0}(X)$ if and only if $u \in \mathcal{D}(\mathcal{E}) \cap C_{0}(X)$.

Lemma 2.6. $\left(\mathcal{E}^{\mu, h}, \mathcal{D}\left(\mathcal{E}^{\mu, h}\right)\right)$ is a regular Dirichlet form on $L^{2}\left(X ; h^{2} m\right)$.

Proof. We see from Lemma 2.5 that $\mathcal{D}\left(\mathcal{E}^{\mu, h}\right) \cap C_{0}(X)$ is dense in $C_{0}(X)$ with respect to the uniform norm.

Suppose $v \in \mathcal{D}\left(\mathcal{E}^{\mu, h}\right)$. Then by the definition of $\mathcal{D}\left(\mathcal{E}^{\mu, h}\right), h v \in \mathcal{D}\left(\mathcal{E}^{\mu}\right)$ and by the regularity of $(\mathcal{E}, \mathcal{D}(\mathcal{E}))$ there exists a sequence $\left\{\varphi_{n}\right\} \subset \mathcal{D}(\mathcal{E}) \cap C_{0}(X)$ such that $\mathcal{E}^{\mu}\left(h v-\varphi_{n}, h v-\varphi_{n}\right)$ converges to 0 as $n \rightarrow \infty$. Hence, $\varphi_{n} / h \in$ $\mathcal{D}\left(\mathcal{E}^{\mu, h}\right) \cap C_{0}(X)$ and

$$
\mathcal{E}^{\mu, h}\left(v-\frac{\varphi_{n}}{h}, v-\frac{\varphi_{n}}{h}\right)=\mathcal{E}^{\mu}\left(h v-\varphi_{n}, h v-\varphi_{n}\right) \rightarrow 0 \quad \text { as } n \rightarrow \infty,
$$

which implies the regularity of $\left(\mathcal{E}^{\mu, h}, \mathcal{D}\left(\mathcal{E}^{\mu, h}\right)\right)$. 
Let us denote by $\mathbf{M}^{\mu, h}$ the Hunt process generated by the regular Dirichlet form $\left(\mathcal{E}^{\mu, h}, \mathcal{D}\left(\mathcal{E}^{\mu, h}\right)\right)$. By the definition of $\left(\mathcal{E}^{\mu, h}, \mathcal{D}\left(\mathcal{E}^{\mu, h}\right)\right)$, the semigroup $\left\{p_{t}^{h}\right\}$ of $\mathbf{M}^{\mu, h}$ is $h^{2} m$-symmetric and

$$
p_{t}^{h} f(x)=\frac{1}{h(x)} E_{x}\left(\exp \left(-A_{t}^{\mu}\right) h\left(X_{t}\right) f\left(X_{t}\right)\right), \quad f \in \mathscr{B}_{b}(X) .
$$

The irreducibility of $\mathbf{M}^{\mu, h}$ follows from that of $\mathbf{M}$ because $\exp \left(-A_{t}^{\mu}\right) h\left(X_{t}\right)>$ 0 . Moreover, $\mathbf{M}^{\mu, h}$ satisfies

(LSC) For $\gamma>0$, every $\gamma$-excessive function is lower-semi-continuous. Indeed, let $G_{\gamma}^{\mu, h}$ be the $\gamma$-resolvent of $\mathbf{M}^{\mu, h}$. Then for $g \in \mathscr{B}_{b}(X)$,

$$
\frac{1}{h(x)} G_{\gamma}^{\mu}(g(h \wedge n))(x) \uparrow G_{\gamma}^{\mu, h} g(x), \quad \text { as } n \rightarrow \infty .
$$

The function $G_{\gamma}^{\mu}(g(h \wedge n))$ is continuous on $X$ by the strong Feller property of $p_{t}^{\mu}$ (see [3, Theorem 1.1]), and thus $G_{\gamma}^{\mu, h} g$ is lower-semi-continuous.

Lemma 2.7. For $h \in \mathcal{H}^{+}$suppose that $\left(\mathcal{E}^{\mu, h}, \mathcal{D}\left(\mathcal{E}^{\mu, h}\right)\right)$ is transient. Then $\mathbf{M}^{\mu, h}$ satisfies (LSC). In particular, $G^{\mu, h} g$ is lower-semi-continuous for each positive Borel function $g$.

Let $\mathcal{D}_{e}\left(\mathcal{E}^{\mu, h}\right)$ be the extended Dirichlet space of the regular Dirichlet form $\left(\mathcal{E}^{\mu, h}, \mathcal{D}\left(\mathcal{E}^{\mu, h}\right)\right)$. We define the extended Schrödinger space $\mathcal{D}_{e}\left(\mathcal{E}^{\mu}\right)$ by

$$
\left\{\begin{array}{l}
\mathcal{E}^{\mu}(u, v)=\mathcal{E}^{\mu, h}\left(\frac{u}{h}, \frac{v}{h}\right) \\
\mathcal{D}_{e}\left(\mathcal{E}^{\mu}\right)=\left\{u: \frac{u}{h} \in \mathcal{D}_{e}\left(\mathcal{E}^{\mu, h}\right)\right\} .
\end{array}\right.
$$

We give another definition of the extended Schrödinger space similar to that of the extended Dirichlet form, that is, the family of $m$-measurable functions $u$ such that $|u|<\infty m$-a.e. and there exists an $\mathcal{E}^{\mu}$-Cauchy sequence $\left\{u_{n}\right\}$ of functions in $\mathcal{D}\left(\mathcal{E}^{\mu}\right)$ such that $\lim _{n \rightarrow \infty} u_{n}=u m$-a.e. Denote by $\widetilde{\mathcal{D}}_{e}\left(\mathcal{E}^{\mu}\right)$ this family, and for $u \in \widetilde{\mathcal{D}}_{e}\left(\mathcal{E}^{\mu}\right)$ and the sequence $\left\{u_{n}\right\}$ define

$$
\widetilde{\mathcal{E}}^{\mu}(u, u)=\lim _{n \rightarrow \infty} \mathcal{E}^{\mu}\left(u_{n}, u_{n}\right) .
$$

Then we see that $\left(\widetilde{\mathcal{E}}^{\mu}, \widetilde{\mathcal{D}}_{e}\left(\mathcal{E}^{\mu}\right)\right)$ is well defined.

Lemma 2.8. $\widetilde{\mathcal{D}}_{e}\left(\mathcal{E}^{\mu}\right)=\mathcal{D}_{e}\left(\mathcal{E}^{\mu}\right)$ and $\widetilde{\mathcal{E}}^{\mu}=\mathcal{E}^{\mu}$.

Proof. For any $u \in \mathcal{D}_{e}\left(\mathcal{E}^{\mu}\right)$, the function $u / h$ is in $\mathcal{D}_{e}\left(\mathcal{E}^{\mu, h}\right)$ by definition, and thus there exists an approximating sequence $\left\{\varphi_{n}\right\} \subset \mathcal{D}\left(\mathcal{E}^{\mu, h}\right)$ of $u / h$. Then $\left\{h \varphi_{n}\right\}$ is an $\mathcal{E}^{\mu}$-Cauchy sequence in $\mathcal{D}\left(\mathcal{E}^{\mu}\right)$ such that $\lim _{n \rightarrow \infty} h \varphi_{n}=$ $h \cdot(u / h)=u m$-a.e. Hence, $u$ belongs to $\widetilde{\mathcal{D}}_{e}\left(\mathcal{E}^{\mu}\right)$.

For any $u \in \widetilde{\mathcal{D}}_{e}\left(\mathcal{E}^{\mu}\right)$, there exists a $\mathcal{E}^{\mu}$-Cauchy sequence $\left\{\varphi_{n}\right\}$ in $\mathcal{D}\left(\mathcal{E}^{\mu}\right)$ such that $\lim _{n \rightarrow \infty} \varphi_{n}=u m$-a.e. Then $\left\{\varphi_{n} / h\right\}$ is an $\mathcal{E}^{\mu, h}$-Cauchy sequence in $\mathcal{D}\left(\mathcal{E}^{\mu, h}\right)$ such that $\lim _{n \rightarrow \infty} \varphi_{n} / h=u / h m$-a.e. Hence, $u / h$ is in $\mathcal{D}_{e}\left(\mathcal{E}^{\mu, h}\right)$, and thus $u$ in $\mathcal{D}_{e}\left(\mathcal{E}^{\mu}\right)$. 
We see from Lemma 2.8 that the space $\mathcal{D}_{e}\left(\mathcal{E}^{\mu}\right)$ is independent of $h \in \mathcal{H}^{+}$. We call the space $\mathcal{D}_{e}\left(\mathcal{E}^{\mu}\right)$ an extended Schrödinger space and the sequence $\left\{u_{n}\right\}$ in the definition of $\widetilde{\mathcal{D}}_{e}\left(\mathcal{E}^{\mu}\right)$ an approximating sequence of $u \in \widetilde{\mathcal{D}}_{e}\left(\mathcal{E}^{\mu}\right)$. In the definition of $\widetilde{\mathcal{D}}_{e}\left(\mathcal{E}^{\mu}\right)$, the condition for $\left\{u_{n}\right\}$ being an $\mathcal{E}^{\mu}$-Cauchy sequence can be replaced by $\sup _{n} \mathcal{E}^{\mu}\left(u_{n}, u_{n}\right)<\infty$ :

Lemma 2.9. Let $\left\{u_{n}\right\}$ be a sequence of functions in $\mathcal{D}\left(\mathcal{E}^{\mu}\right)$ such that

$$
\sup _{n} \mathcal{E}^{\mu}\left(u_{n}, u_{n}\right)<\infty, \quad \lim _{n \rightarrow \infty} u_{n}=u \quad \text { m-a.e. }
$$

Then $u$ belongs to $\mathcal{D}_{e}\left(\mathcal{E}^{\mu}\right)$.

Proof. The sequence $\left\{u_{n} / h\right\} \subset \mathcal{D}\left(\mathcal{E}^{\mu, h}\right)$ satisfies

$$
\sup _{n} \mathcal{E}^{\mu, h}\left(\frac{u_{n}}{h}, \frac{u_{n}}{h}\right)<\infty, \quad \lim _{n \rightarrow \infty} \frac{u_{n}}{h}=\frac{u}{h} \quad m \text {-a.e. }
$$

Hence, $u / h$ is in $\mathcal{D}_{e}\left(\mathcal{E}^{\mu, h}\right)$ by [12, Definition 1.6], and so $u$ in $\mathcal{D}_{e}\left(\mathcal{E}^{\mu}\right)$.

\section{Poincaré-type inequalities for Schrödinger forms}

First, we would like to remind the reader an inequality for recurrent Dirichlet forms [6, Theorem 4.8.2(ii)]. A Hunt process $\mathbf{M}=\left(P_{x}, X_{t}\right)$ is said to be Harris recurrent if for any Borel set $B$ with $m(B)>0$

$$
\int_{0}^{\infty} 1_{B}\left(X_{s}\right) d s=\infty, \quad P_{x} \text {-a.s. for any } x \in X .
$$

TheOREm 3.1. Suppose $\mathbf{M}$ is Harris recurrent. Then there exist a nearly Borel finely closed set $B$ with $0<m(B)<\infty$ and a strictly positive function $g$ in $L^{1}(X ; m)$ such that for all $u \in \mathcal{D}_{e}(\mathcal{E})$

$$
\int_{X}\left(u(x)-\frac{1}{m(B)} \int_{B} u d m\right)^{2} g(x) m(d x) \leq \mathcal{E}(u, u) .
$$

We apply this Poincaré-type inequality to Schrödinger forms.

Theorem 3.2. Assume that $\left(\mathcal{E}^{\mu, h}, \mathcal{D}\left(\mathcal{E}^{\mu, h}\right)\right)$ is recurrent for $h \in \mathcal{H}^{+}$. Then there exist a nearly Borel finely closed set $B$ with $0<m(B)<\infty$ and a strictly positive function $g$ in $L^{1}\left(X ; h^{2} m\right)$ such that for any $u \in \mathcal{D}_{e}\left(\mathcal{E}^{\mu}\right)$,

$$
\int_{X}\left(u-D_{h}(u) h\right)^{2} g(x) m(d x) \leq \mathcal{E}^{\mu}(u, u),
$$

where $D_{h}(u)=\left\|h 1_{B}\right\|_{2}^{-2} \int_{B} u(x) h(x) m(d x)$.

Proof. First, remark that an irreducible, recurrent, symmetric Hunt process satisfying (AC) is Harris recurrent [6, Lemma 4.8.1]. Since $\mathbf{M}^{\mu, h}$ is an $h^{2} m$-symmetric Hunt process with transition density:

$$
p^{\mu, h}(t, x, y)=\frac{p^{\mu}(t, x, y)}{h(x) h(y)}
$$


with respect to $h^{2} m$, it is Harris recurrent. Hence, we can apply Theorem 3.1 to $\mathbf{M}^{\mu, h}$; there exist a Borel set $B$ with $\int_{B} h^{2}(x) m(d x)<\infty$ and a strictly positive bounded $g \in L^{1}\left(h^{2} m\right)$ such that for all $u \in \mathcal{D}_{e}\left(\mathcal{E}^{\mu, h}\right)$,

$$
\begin{aligned}
& \int_{X}\left|u(x)-\left(\int_{B} h^{2}(x) m(d x)\right)^{-1} \int_{B} u(x) h^{2}(x) m(d x)\right|^{2} g(x) h^{2}(x) m(d x) \\
& \leq \mathcal{E}^{\mu, h}(u, u) .
\end{aligned}
$$

Let $L(u)=\left(\int_{B} h^{2}(x) m(d x)\right)^{-1} \int_{B} u(x) h^{2}(x) m(d x)$. We then know

$$
\int_{X}|u(x)-L(u)|^{2} g(x) h^{2}(x) m(d x) \leq \mathcal{E}^{\mu}(h u, h u) .
$$

Put $v=h u$. Then $v$ is an element of $\mathcal{D}_{e}\left(\mathcal{E}^{\mu}\right)$ and (3.3) leads us to

$$
\int_{X}\left|v(x)-D_{h}(v) h(x)\right|^{2} g(x) m(d x) \leq \mathcal{E}^{\mu}(v, v),
$$

where

$$
D_{h}(v)=L(v / h)=\left\|h 1_{B}\right\|_{2}^{-2} \int_{B} v h d m .
$$

We, in addition, make the next assumption:

(PC) The transition probability density $p(t, x, y)$ is strictly positive and jointly continuous on $X \times X$.

Denote by $\mathscr{B}_{b, 0}^{+}(X)$ the set of non-negative functions on $X$ with compact support.

Lemma 3.3. Assume (PC). Then the 1-resolvent density of the time changed process by $\int_{0}^{t} \varphi\left(X_{s}\right) d s, \varphi \in \mathscr{B}_{b, 0}^{+}(X)$, is bounded from below by a positive constant on the fine support of $\varphi \cdot m$,

$$
\inf _{(x, y) \in F \times F} \check{G}_{1}(x, y)>0,
$$

where $\check{G}_{1}$ is the 1-resolvent density of the time changed process and $F$ is the fine support of $\varphi \cdot m$.

Proof. Put $\mu=\varphi \cdot m$ and define $\tau_{t}=\inf \left\{s>0: A_{s}^{\mu}>t\right\}\left(A_{t}^{\mu}=\int_{0}^{t} \varphi\left(X_{s}\right) d s\right)$. Then

$$
\begin{aligned}
\check{G}_{1} f(x) & =E_{x}\left(\int_{0}^{\infty} e^{-t} f\left(X_{\tau_{t}}\right) d t\right) \\
& =E_{x}\left(\int_{0}^{\infty} e^{-A_{t}^{\mu}} f\left(X_{t}\right) d A_{t}^{\mu}\right)=G^{\mu}(f \cdot \mu), \quad x \in F,
\end{aligned}
$$

and so $\check{G}_{1}(x, y)=G^{\mu}(x, y)$ for $(x, y) \in F \times F$. Noting that $p_{t}^{\mu} f(x) \geq$ $E_{x}\left(\exp (-\beta t) f\left(X_{t}\right)\right),\|\varphi\|_{\infty}=\beta$, we have $G^{\mu}(x, y) \geq G_{\beta}(x, y)$. It follows from 
(PC) that $G_{\beta}(x, y)$ is strictly positive and lower-semi-continuous. Therefore, we have

$$
\inf _{(x, y) \in F \times F} \check{G}_{1}(x, y) \geq \inf _{(x, y) \in F \times F} G_{\beta}(x, y)>0 .
$$

Through Lemma 3.3, we can follow the argument in [6, Section 4.8] and extend the inequality (3.2) as follows: for any $\varphi \in \mathscr{B}_{b, 0}^{+}(X)$ with $\int_{X} \varphi d m \neq 0$ there exist a strictly positive continuous function $g$ in $L^{1}(X ; m)$ such that for any $u \in \mathcal{D}(\mathcal{E})$,

$$
\int_{X}(u-L(u))^{2} g d m \leq \mathcal{E}(u, u), \quad L(u)=\int_{X} u \varphi d m / \int_{X} \varphi d m .
$$

For $\varphi=\varphi^{+}-\varphi^{-} \in \mathscr{B}_{b, 0}^{+}(X)-\mathscr{B}_{b, 0}^{+}(X)$, put

$$
L^{+}(u)=\frac{\int_{X} u \varphi^{+} d m}{\int_{X} \varphi^{+} d m}, \quad L^{-}(u)=\frac{\int_{X} u \varphi^{-} d m}{\int_{X} \varphi^{-} d m}
$$

and

$$
c^{+}=\frac{\int_{X} \varphi^{+} d m}{\int_{X} \varphi d m}, \quad c^{-}=\frac{\int_{X} \varphi^{-} d m}{\int_{X} \varphi d m} .
$$

Note that

$$
L(u)=c^{+} L^{+}(u)-c^{-} L^{-}(u), \quad c^{+}-c^{-}=1
$$

and

$$
\begin{aligned}
u-L(u) & =\left(c^{+}-c^{-}\right) u-\left(c^{+} L^{+}(u)-c^{-} L^{-}(u)\right) \\
& =c^{+}\left(u-L^{+}(u)\right)-c^{-}\left(u-L^{-}(u)\right) .
\end{aligned}
$$

We then have

$$
\begin{aligned}
\int_{X} u^{2} g d m \leq & 2 \int_{X}(u-L(u))^{2} g d m+2 L(u)^{2} \int_{X} g d m \\
\leq & 4\left(c^{+}\right)^{2} \int_{X}\left(u-L^{+}(u)\right)^{2} g d m+4\left(c^{-}\right)^{2} \int_{X}\left(u-L^{-}(u)\right)^{2} g d m \\
& +2 \frac{\int_{X} g d m}{\left(\int_{X} \varphi d m\right)^{2}}\left(\int_{X} u \varphi d m\right)^{2} .
\end{aligned}
$$

Letting

$$
C=4\left(\left(c^{+}\right)^{2}+\left(c^{-}\right)^{2}\right) \vee\left(2 \frac{\int_{X} g d m}{\left(\int_{X} \varphi d m\right)^{2}}\right),
$$

we see from (3.5) that

$$
\frac{1}{C} \int_{X} u^{2} g d m \leq \mathcal{E}(u, u)+C\left(\int_{X} u \varphi d m\right)^{2} .
$$

Hence, we have the next corollary. 
Corollary 3.4. Assume $\mathbf{M}$ satisfies $(\mathrm{PC})$. If $(\mathcal{E}, \mathcal{D}(\mathcal{E}))$ is recurrent, then for any $\varphi \in \mathscr{B}_{b, 0}(X)$ with $\int_{X} \varphi d m \neq 0$ there exists a strictly positive function $g$ in $L^{1}(X ; m)$ and a positive constant $C$ such that

$$
\frac{1}{C} \int_{X} u^{2} g d m \leq \mathcal{E}(u, u)+C\left(\int_{X} u \varphi d m\right)^{2}, \quad u \in \mathcal{D}_{e}(\mathcal{E}) .
$$

Applying Corollary 3.4 to $\left(\mathcal{E}^{\mu, h}, \mathcal{D}\left(\mathcal{E}^{\mu, h}\right)\right)$, we have

Corollary 3.5. Assume $\mathbf{M}$ satisfies $(\mathrm{PC})$. If $\left(\mathcal{E}^{\mu}, \mathcal{D}\left(\mathcal{E}^{\mu}\right)\right)$ is critical, then for any $\varphi \in \mathscr{B}_{b, 0}(X)$ with $\int_{X} \varphi h d m \neq 0$ there exist a strictly positive bounded function $g$ and a positive constant $C$ such that

$$
\frac{1}{C} \int_{X} u^{2} g d m \leq \mathcal{E}^{\mu}(u, u)+C\left(\int_{X} u \varphi d m\right)^{2}, \quad v \in \mathcal{D}_{e}\left(\mathcal{E}^{\mu}\right) .
$$

Proof. By Corollary 3.4, for any $\phi \in \mathscr{B}_{b, 0}(X)$ with $\int_{X} \phi h^{2} d m \neq 0$ there exists a function $g$ in $L^{1}\left(X ; h^{2} m\right)$ a positive constant $C$ such that

$$
\frac{1}{C} \int_{X} v^{2} g h^{2} d m \leq \mathcal{E}^{\mu, h}(v, v)+C\left(\int_{X} v \phi h^{2} d m\right)^{2}, \quad u \in \mathcal{D}_{e}\left(\mathcal{E}^{\mu, h}\right) .
$$

Putting $u=v h \in \mathcal{D}_{e}\left(\mathcal{E}^{\mu}\right)$ and $\varphi=\phi h \in \mathscr{B}_{b, 0}(X)$, we have (3.7). Note that $\int_{X} \phi h^{2} d m \neq 0$ is replaced by $\int_{X} \varphi h d m \neq 0$.

The inequality (3.7) is established in [11] for critical Schrödinger differential operators. We would like to emphasize that (3.7) is derived from (3.2).

Lemma 3.6. If $\left(\mathcal{E}^{\mu, h}, \mathcal{D}\left(\mathcal{E}^{\mu, h}\right)\right)$ is transient for $h \in \mathcal{H}^{+}$, then there exists a strictly positive continuous function $g$ such that $\left\|G^{\mu, h}(g)\right\|_{\infty} \leq 1$.

Proof. First, note that $\mathbf{M}^{\mu, h}$ satisfies (I) and (LSC). Applying [7, Corollary (2.3)], we can show by the same argument as in [14, Lemma 2.4] that there exists a strictly positive continuous function $f$ such that the measure $f h^{2} m$ belongs to the Green-tight Kato class associated with $\mathbf{M}^{\mu, h}$, and consequently $\left\|G^{\mu, h}(f)\right\|_{\infty}<\infty$ by Chen [2, Proposition 2.2]. Hence, $g:=f /\left\|G^{\mu, h}(f)\right\|_{\infty}$ is a desired one.

Theorem 3.7. Let $h \in \mathcal{H}^{+}$and suppose $\left(\mathcal{E}^{\mu, h}, \mathcal{D}\left(\mathcal{E}^{\mu, h}\right)\right)$ is transient. Then there exists a strictly positive continuous function $g$ such that for $u \in \mathcal{D}_{e}\left(\mathcal{E}^{\mu}\right)$

$$
\int_{X} u^{2} g d m \leq \mathcal{E}^{\mu}(u, u) .
$$

Proof. Let $g$ be the function in Lemma 3.6. We see from (2.3) that

$$
\int_{X} v^{2} g h^{2} d m \leq \mathcal{E}^{\mu, h}(v, v), \quad v \in \mathcal{D}_{e}\left(\mathcal{E}^{\mu, h}\right) .
$$


Putting $u=h v \in \mathcal{D}_{e}\left(\mathcal{E}^{\mu}\right)$, we obtain

$$
\int_{X} u^{2} g d m \leq \mathcal{E}^{\mu, h}\left(\frac{u}{h}, \frac{u}{h}\right)=\mathcal{E}^{\mu}(u, u) .
$$

We would like to emphasize that the inequalities $(3.2)$, (3.9) hold for all elements in the extended Schrödinger space $\mathcal{D}_{e}\left(\mathcal{E}^{\mu}\right)$. In particular, this extension is crucial for defining the criticality of Schrödinger forms.

\section{Criticality and subcriticality}

LEMmA 4.1. Suppose that $\left(\mathcal{E}^{\mu, h}, \mathcal{D}\left(\mathcal{E}^{\mu, h}\right)\right)$ is recurrent for a function $h$ in $\mathcal{H}^{+}$. Then the function $h$ belongs to $\mathcal{D}_{e}\left(\mathcal{E}^{\mu}\right)$ and $\mathcal{E}^{\mu}(h, h)=0$.

Proof. We see from [6, Theorem 1.6.3] that the constant function 1 belongs to $\mathcal{D}_{e}\left(\mathcal{E}^{\mu, h}\right)$ if and only if $\left(\mathcal{E}^{\mu, h}, \mathcal{D}\left(\mathcal{E}^{\mu, h}\right)\right)$ is recurrent. Hence, there exists an approximating sequence $\left\{\varphi_{n}\right\} \subset \mathcal{D}\left(\mathcal{E}^{\mu, h}\right)$ of 1 . Then $\left\{h \varphi_{n}\right\} \subset \mathcal{D}(\mathcal{E})$ becomes an approximating sequence of $h$, and thus this lemma follows from the definition of extended Schrödinger form.

LEMMA 4.2. Suppose $\mathcal{H}^{+}$is not empty. If $\left(\mathcal{E}^{\mu, h}, \mathcal{D}\left(\mathcal{E}^{\mu, h}\right)\right)$ is transient for some $h \in \mathcal{H}^{+}$, then so is for any $h \in \mathcal{H}^{+}$.

Proof. First, note that by $(\mathrm{I}),\left(\mathcal{E}^{\mu, h}, \mathcal{D}\left(\mathcal{E}^{\mu, h}\right)\right)$ is either transient or recurrent. Suppose that for $h$ and $\widetilde{h}$ in $\mathcal{H}^{+},\left(\mathcal{E}^{\mu, h}, \mathcal{D}\left(\mathcal{E}^{\mu, h}\right)\right)$ and $\left(\mathcal{E}^{\mu, \widetilde{h}}, \mathcal{D}\left(\mathcal{E}^{\mu, \widetilde{h}}\right)\right)$ are transient and recurrent respectively. Then it follows from Theorem 3.7 there exists $g>0$ such that

$$
\int_{X} u^{2} g d m \leq \mathcal{E}^{\mu}(u, u), \quad \text { for } u \in \mathcal{D}_{e}\left(\mathcal{E}^{\mu}\right) .
$$

Since $\widetilde{h}$ belongs to $\mathcal{D}_{e}(\mathcal{E})$,

$$
\int_{X} \widetilde{h}^{2} g d m \leq \mathcal{E}^{\mu}(\widetilde{h}, \widetilde{h})=0
$$

by (4.1) and Lemma 4.1. Consequently, $\widetilde{h}=0 m$-a.e., which is contradictory.

LEMMA 4.3. Suppose $\mathcal{H}^{+}$is not empty. If $\left(\mathcal{E}^{\mu, h}, \mathcal{D}\left(\mathcal{E}^{\mu, h}\right)\right)$ is recurrent for some $h \in \mathcal{H}^{+}$, then each $\widetilde{h} \in \mathcal{H}^{+}$can be written as $\widetilde{h}=$ ch. Here c is a positive constant.

Proof. By Theorem 3.2 and Lemma 4.1,

$$
\int_{X}\left(\widetilde{h}-D_{h}(\widetilde{h}) h\right)^{2} g(x) m(d x) \leq \mathcal{E}^{\mu}(\widetilde{h}, \widetilde{h})=0,
$$

and thus $\widetilde{h}=c h, c=D_{h}(\widetilde{h})$. 
On account of Lemma 4.2 and Lemma 4.3 we define the subcriticality, criticality and supercriticality for Schrödinger forms as follows.

Definition 4.4. Let $\mu \in \mathcal{K}_{\text {loc }}-\mathcal{K}$ and $\left(\mathcal{E}^{\mu}, \mathcal{D}\left(\mathcal{E}^{\mu}\right)\right)$ the Schrödinger form defined by $(2.4)$.

(1) $\left(\mathcal{E}^{\mu}, \mathcal{D}\left(\mathcal{E}^{\mu}\right)\right)$ is said to be subcritical if $\left(\mathcal{E}^{\mu, h}, \mathcal{D}\left(\mathcal{E}^{\mu, h}\right)\right)$ is transient for some $h \in \mathcal{H}^{+}$.

(2) $\left(\mathcal{E}^{\mu}, \mathcal{D}\left(\mathcal{E}^{\mu}\right)\right)$ is said to be critical if $\left(\mathcal{E}^{\mu, h}, \mathcal{D}\left(\mathcal{E}^{\mu, h}\right)\right)$ is recurrent for some $h \in \mathcal{H}^{+}$.

(3) $\left(\mathcal{E}^{\mu}, \mathcal{D}\left(\mathcal{E}^{\mu}\right)\right)$ is said to be supercritical if $\mathcal{H}^{+}=\emptyset$.

Remark 4.5. Suppose $\mathcal{H}^{+}$is not empty. Then for $h \in \mathcal{H}^{+}$,

$$
\mathcal{E}^{\mu}(u, u)=\mathcal{E}^{\mu, h}\left(\frac{u}{h}, \frac{u}{h}\right) \geq 0 .
$$

Hence, if $\mathcal{E}^{\mu}$ is not positive definite, then $\mathcal{H}^{+}$is empty, i.e., $\left(\mathcal{E}^{\mu}, \mathcal{D}\left(\mathcal{E}^{\mu}\right)\right)$ is supercritical by definition.

A Dirichlet form $(\mathcal{E}, \mathcal{D}(\mathcal{E}))$ is said to be local, if $\mathcal{E}(u, v)=0$ for any $u, v \in$ $\mathcal{D}(\mathcal{E})$ with $\operatorname{supp}[u] \cap \operatorname{supp}[v]=\emptyset$.

Lemma 4.6. If $(\mathcal{E}, \mathcal{D}(\mathcal{E}))$ is local, then so is $\left(\mathcal{E}^{\mu, h}, \mathcal{D}\left(\mathcal{E}^{\mu, h}\right)\right)$.

Proof. Since

$$
\operatorname{supp}[u] \cap \operatorname{supp}[v]=\emptyset \quad \Longleftrightarrow \quad \operatorname{supp}[h u] \cap \operatorname{supp}[h v]=\emptyset,
$$

this lemma follows from the definition of $\left(\mathcal{E}^{\mu, h}, \mathcal{D}\left(\mathcal{E}^{\mu, h}\right)\right)$.

Assume that $(\mathcal{E}, \mathcal{D}(\mathcal{E}))$ is local. We set

$$
\widetilde{\mathcal{H}}^{+}=\left\{h \in \mathcal{D}_{\text {loc }}(\mathcal{E}) \cap C(X): \mathcal{E}^{\mu}(h, \varphi) \geq 0 \text { for any } \varphi \in \mathcal{D}(\mathcal{E}) \cap C_{0}^{+}(X)\right\} .
$$

Lemma 4.7. If $(\mathcal{E}, \mathcal{D}(\mathcal{E}))$ is local, then

$$
\mathcal{H}^{+}=\widetilde{\mathcal{H}}^{+} .
$$

Proof. (i) $\left(\mathcal{H}^{+} \subset \widetilde{\mathcal{H}}^{+}\right)$. Let $\varphi \in \mathcal{D}(\mathcal{E}) \cap C_{0}(X)$. Then

$$
\mathcal{E}^{\mu, h}\left(u, \frac{\varphi}{h}\right)=\mathcal{E}^{\mu}(h u, \varphi), \quad u \in \mathcal{D}\left(\mathcal{E}^{\mu, h}\right) .
$$

By Lemma 4.6, the Dirichlet form $\mathcal{E}^{\mu, h}(u, v)$ is written as

$$
\mathcal{E}^{\mu, h}(u, v)=\frac{1}{2} \int_{X} d \widetilde{\mu}_{\langle u, v\rangle}^{c}+\int_{X} u v d \widetilde{k},
$$

where $\widetilde{\mu}_{\langle u, v\rangle}^{c}$ is the energy measure of $u$ and $v$ associated with the strongly local part of $\mathcal{E}^{\mu, h}$ and $\widetilde{k}$ is the killing measure (see [6, Theorem 5.3.1]). 
For $u \in \mathcal{D}(\mathcal{E}) \cap C_{0}(X)$ such that $u=1$ on $\operatorname{supp}[\varphi]$,

$$
\begin{aligned}
\mathcal{E}^{\mu, h}\left(u, \frac{\varphi}{h}\right) & =\frac{1}{2} \int_{X} d \widetilde{\mu}_{\langle u, \varphi / h\rangle}^{c}+\int_{X} u \frac{\varphi}{h} d \widetilde{k} \\
& =\frac{1}{2} \int_{X} d \widetilde{\mu}_{\langle 1, \varphi / h\rangle}^{c}+\int_{X} \frac{\varphi}{h} d \widetilde{k}=\int_{X} \frac{\varphi}{h} d \widetilde{k} .
\end{aligned}
$$

In addition,

$\mathcal{E}^{\mu}(h u, \varphi)=\frac{1}{2} \int_{X} d \mu_{\langle h u, \varphi\rangle}^{c}+\int_{X} h u \varphi d \mu=\frac{1}{2} \int_{X} d \mu_{\langle h, \varphi\rangle}^{c}+\int_{X} h \varphi d \mu=\mathcal{E}^{\mu}(h, \varphi)$, where $\mu_{\langle u, v\rangle}^{c}$ is the energy measure of the strongly local part of $\mathcal{E}$. Hence for any non-negative function $\varphi$ in $\mathcal{D}(\mathcal{E}) \cap C_{0}(X)$

$$
\mathcal{E}^{\mu}(h, \varphi)=\mathcal{E}^{\mu}(h u, \varphi)=\mathcal{E}^{\mu, h}(u, \varphi / h)=\int_{X} \frac{\varphi}{h} d \widetilde{k} \geq 0 .
$$

(ii) $\left(\mathcal{H}^{+} \supset \widetilde{\mathcal{H}}^{+}\right)$. Let $\mathcal{L}=\mathcal{D}(\mathcal{E}) \cap C_{0}(X)$. Then $\mathcal{L}$ is a Stone vector lattice, if $f, g \in \mathcal{L}$, then $f \vee g \in \mathcal{L}, f \wedge 1 \in \mathcal{L}$. For $h \in \widetilde{\mathcal{H}}^{+}$, define the functional $I$ by

$$
I(\varphi)=\mathcal{E}^{\mu}(h, \varphi), \quad \varphi \in \mathcal{L} .
$$

Then $I(\varphi)$ is a pre-integral, that is, $I\left(\varphi_{n}\right) \downarrow 0$ whenever $\varphi_{n} \in \mathcal{L}$ and $\varphi_{n}(x) \downarrow 0$ for all $x \in X$. Indeed, let $\psi \in \mathcal{D}(\mathcal{E}) \cap C_{0}(X)$ such that $\psi=1$ on $\operatorname{supp}\left[\varphi_{1}\right]$. Then $\varphi_{n} \leq\left\|\varphi_{n}\right\|_{\infty} \psi$ and

$$
I\left(\varphi_{n}\right) \leq\left\|\varphi_{n}\right\|_{\infty} \cdot I(\psi) \downarrow 0, \quad n \rightarrow \infty .
$$

Noting that the smallest $\sigma$-field generated by $\mathcal{L}$ is identical with the Borel $\sigma$-field, we know that there exists a Borel measure $\nu$ such that

$$
I(\varphi)=\int_{X} \varphi d \nu
$$

[5, Theorem 4.5.2]. Moreover, we see that the measure $\nu$ is smooth. Indeed, let $K$ be a compact set of zero capacity, and take a relatively compact open set $D$ such that $K \subset D$. Then there exists a sequence $\left\{\varphi_{n}\right\} \subset \mathcal{D}(\mathcal{E}) \cap C_{0}(D)$ such that $\varphi_{n} \geq 1$ on $K$ and $\mathcal{E}_{1}\left(\varphi_{n}, \varphi_{n}\right) \rightarrow 0$ as $n \rightarrow \infty$. For $\psi \in \mathcal{D}(\mathcal{E}) \cap C_{0}(X)$ with $\psi=1$ on $D$,

$$
I\left(\varphi_{n}\right)=\mathcal{E}^{\mu}\left(h \psi, \varphi_{n}\right) \leq \mathcal{E}^{\mu}(h \psi, h \psi)^{1 / 2} \cdot \mathcal{E}^{\mu}\left(\varphi_{n}, \varphi_{n}\right)^{1 / 2} .
$$

Since

$$
\int_{X} \varphi_{n}^{2} d \mu \leq\left\|G_{1} \mu\right\|_{\infty} \cdot \mathcal{E}_{1}\left(\varphi_{n}, \varphi_{n}\right) \rightarrow 0, \quad n \rightarrow \infty
$$

$\mathcal{E}^{\mu}\left(\varphi_{n}, \varphi_{n}\right) \rightarrow 0$ as $n \rightarrow \infty$. Consequently,

$$
\nu(K) \leq \int_{X} \varphi_{n} d \nu=I\left(\varphi_{n}\right) \leq \mathcal{E}^{\mu}(h \psi, h \psi)^{1 / 2} \cdot \mathcal{E}^{\mu}\left(\varphi_{n}, \varphi_{n}\right)^{1 / 2} \rightarrow 0 .
$$


The equation (4.3) is equivalent to

$$
\mathcal{E}(h, \varphi)=-\int_{X} \varphi h d \mu+\int_{X} \varphi d \nu=\int_{X} \varphi(-h d \mu+d \nu) .
$$

On account of $[6$, Theorem 5.5.5], we have

$$
h\left(X_{t}\right)=h\left(X_{0}\right)+M_{t}^{[h]}+\int_{0}^{t} h\left(X_{s}\right) d A_{s}^{\mu}-A_{t}^{\nu}, \quad P_{x^{-}} \text {a.s. q.e. } x .
$$

Hence, by Itô's formula

$$
\begin{aligned}
e^{-A_{t}^{\mu}} h\left(X_{t}\right)= & h\left(X_{0}\right)+\int_{0}^{t} e^{-A_{s}^{\mu}} d M_{s}^{[h]}+\int_{0}^{t} e^{-A_{s}^{\mu}} h\left(X_{s}\right) d A_{s}^{\mu} \\
& -\int_{0}^{t} e^{-A_{s}^{\mu}} d A_{s}^{\nu}-\int_{0}^{t} e^{-A_{s}^{\mu}} h\left(X_{s}\right) d A_{s}^{\mu} \\
= & h\left(X_{0}\right)+\int_{0}^{t} e^{-A_{s}^{\mu}} d M_{s}^{[h]}-\int_{0}^{t} e^{-A_{s}^{\mu}} d A_{s}^{\nu} \quad P_{x^{-a . s . ~}} \text { q.e. } x .
\end{aligned}
$$

Since $\int_{0}^{t} e^{-A_{s}^{\mu}} d M_{s}^{[h]}$ is a local martingale, there exists a sequence $\left\{\sigma_{n}\right\}$ such that $\lim _{n \rightarrow \zeta} \sigma_{n}=\infty$ and $\int_{0}^{\sigma_{n} \wedge t} e^{-A_{s}^{\mu}} d M_{s}^{[h]}$ is a martingale. Hence,

$$
E_{x}\left(e^{-A_{\sigma_{n} \wedge t}^{\mu} h}\left(X_{\sigma_{n} \wedge t}\right)\right) \leq h(x) \quad \text { q.e. } x
$$

and thus, by Fatou's lemma, $p_{t}^{\mu} h(x) \leq h(x)$ for q.e. $x$. Note that $p_{t}^{\mu} h$ is lowersemi-continuous. Then the set $B:=\left\{x \in X: p_{t}^{\mu} h(x)>h(x)\right\}$ is open and of zero capacity, and so empty.

\section{Existence of superharmonic functions}

In this section, we discuss the existence of a function in $\mathcal{H}^{+}$if $\left(\mathcal{E}^{\mu}, \mathcal{D}\left(\mathcal{E}^{\mu}\right)\right)$ is subcritical or critical. Assume that $\mu$ is non-trivial, $\mu^{-} \not \equiv 0$. Define

$$
\lambda(\mu):=\inf \left\{\mathcal{E}(u, u)+\int_{X} u^{2} d \mu^{+}: u \in \mathcal{D}(\mathcal{E}), \int_{X} u^{2} d \mu^{-}=1\right\} .
$$

Owing to [15, Lemma 2.2], we have the following lemma.

LEMMA 5.1.

$$
\lambda(\mu) \geq 1 \Longleftrightarrow \inf \left\{\mathcal{E}(u, u)+\int_{X} u^{2} d \mu: \int_{X} u^{2} d m=1\right\} \geq 0 .
$$

For $\nu \in \mathcal{K}_{\text {loc }}$, let $\mathbf{M}^{\nu}=\left(P_{x}^{\nu}, X_{t}\right)$ be the subprocess of $\mathbf{M}$ killing at rate $\nu$,

$$
P_{x}^{\nu}(\Lambda ; t<\zeta)=E_{x}\left(\exp \left(-A_{t}^{\nu}\right) 1_{\Lambda} ; t<\zeta\right), \quad \Lambda \in \mathscr{F}_{t} .
$$

Let $\left\{p_{t}^{\nu}\right\}_{t \geq 0}$ and $\left\{G_{\beta}^{\nu}\right\}_{\beta \geq 0}$ be the semigroup and resolvent of $\mathbf{M}^{\nu}$,

$$
p_{t}^{\nu} f(x)=E_{x}\left(e^{-A_{t}^{\nu}} f\left(X_{t}\right)\right), \quad G_{\beta}^{\nu} f(x)=E_{x}\left(\int_{0}^{\zeta} e^{-\beta t-A_{t}^{\nu}} f\left(X_{t}\right) d t\right) .
$$


We write $G^{\nu}$ for $G_{0}^{\nu}$ simply. Denote by $G^{\nu}(x, y)$ the Green kernel of $\mathbf{M}^{\nu}$, the integral kernel of $G^{\nu}$,

$$
E_{x}\left(\int_{0}^{\zeta} e^{-A_{t}^{\nu}} f\left(X_{t}\right) d t\right)=\int_{X} G^{\nu}(x, y) f(y) d m(y) .
$$

In the sequel, we suppose that $\left(\mathcal{E}^{\mu^{+}}, \mathcal{D}\left(\mathcal{E}^{\mu^{+}}\right)\right)$is transient and $\mu^{-}$is a nontrivial measure in $\mathcal{K}_{\infty}\left(G^{\mu^{+}}\right)$, the set of Green-tight measures associated with $\mathbf{M}^{\mu^{+}}$.

5.1. Subcritical case. In this subsection, we treat the case that $\lambda(\mu)>1$. Let $g^{\mu}(x)$ be a so-called gauge function defined by

$$
g^{\mu}(x)=E_{x}^{\mu^{+}}\left(e^{A_{\zeta}^{\mu^{-}}}\right)
$$

Then it is known in [2, Theorem 5.1] that $g^{\mu}$ is bounded function if and only if $\lambda(\mu)>1$.

Lemma 5.2. The function $g^{\mu}$ is $p_{t}^{\mu}$-excessive, $p_{t}^{\mu} g^{\mu}(x) \uparrow g^{\mu}(x)$ as $t \downarrow 0$.

Proof. By the Markov property of $\mathbf{M}^{\mu^{+}}$

$$
\begin{aligned}
E_{x}\left(e^{-A_{t}^{\mu}} g^{\mu}\left(X_{t}\right)\right) & =E_{x}\left(e^{-A_{t}^{\mu}} g^{\mu}\left(X_{t}\right) ; t<\zeta\right)=E_{x}^{\mu^{+}}\left(e^{A_{t}^{\mu^{-}}} g^{\mu}\left(X_{t}\right) ; t<\zeta\right) \\
& =E_{x}^{\mu^{+}}\left(e^{A_{t}^{\mu^{-}}} E_{X_{t}}^{\mu^{+}}\left(e^{A_{\zeta}^{\mu^{-}}}\right) ; t<\zeta\right) \\
& =E_{x}^{\mu^{+}}\left(E_{x}^{\mu^{+}}\left(e^{A_{t}^{\mu^{-}}+A_{\zeta}^{\mu^{-}}\left(\theta_{t}\right)} 1_{\{t<\zeta\}} \mid \mathscr{F}_{t}\right)\right) .
\end{aligned}
$$

The right-hand side equals $E_{x}^{\mu^{+}}\left(e^{A_{\zeta}^{\mu^{-}}} ; t<\zeta\right)$ because $A_{t}^{\mu^{-}}+A_{\zeta}^{\mu^{-}}\left(\theta_{t}\right)=$ $A_{\zeta}^{\mu^{-}}$on $\{t<\zeta\}$. Therefore, as $t \downarrow 0$

$$
p_{t}^{\mu} g^{\mu}(x)=E_{x}^{\mu^{+}}\left(e^{A_{\zeta}^{\mu-}} ; t<\zeta\right) \uparrow E_{x}^{\mu^{+}}\left(e^{A_{\zeta}^{\mu-}}\right)=g^{\mu}(x) .
$$

LEMMA 5.3. It holds that

$$
g^{\mu}(x)=1+G^{\mu^{+}}\left(g^{\mu} \mu^{-}\right)(x) .
$$

Proof. Define a uniformly integrable martingale $M_{t}$ by

$$
M_{t}=E_{x}^{\mu^{+}}\left(e^{A_{\zeta}^{\mu^{-}}} \mid \mathscr{F}_{t}\right) .
$$

Then

$$
\int_{0}^{t} g^{\mu}\left(X_{s}\right) d A_{s}^{\mu^{-}}=\int_{0}^{t} e^{-A_{s}^{\mu^{-}}} M_{s} d A_{s}^{\mu^{-}}, \quad t<\zeta
$$

because

$$
\begin{aligned}
e^{-A_{t}^{\mu^{-}} M_{t} 1_{\{t<\zeta\}}} & =e^{-A_{t}^{\mu^{-}}} E_{x}^{\mu^{+}}\left(e^{A_{\zeta}^{\mu^{-}}} 1_{\{t<\zeta\}} \mid \mathscr{F}_{t}\right) \\
& =e^{-A_{t}^{\mu^{-}}} E_{x}^{\mu^{+}}\left(e^{A_{t}^{\mu^{-}}+A_{\zeta}^{\mu^{-}}\left(\theta_{t}\right)} 1_{\{t<\zeta\}} \mid \mathscr{F}_{t}\right) \\
& =E_{X_{t}}^{\mu^{+}}\left(e^{A_{\zeta}^{\mu^{-}}}\right) 1_{\{t<\zeta\}}=g^{\mu}\left(X_{t}\right) 1_{\{t<\zeta\}} .
\end{aligned}
$$


By Itô's formula,

$$
e^{-A_{t}^{\mu^{-}}} M_{t}=M_{0}+\int_{0}^{t} e^{-A_{s}^{\mu^{-}}} d M_{s}-\int_{0}^{t} e^{-A_{s}^{\mu^{-}}} M_{s} d A_{s}^{\mu^{-}}
$$

and thus

$$
E_{x}^{\mu^{+}}\left(M_{0}\right)=E_{x}^{\mu^{+}}\left(e^{-A_{\zeta}^{\mu^{-}}} M_{\zeta}\right)+E_{x}^{\mu^{+}}\left(\int_{0}^{\zeta} e^{-A_{s}^{\mu^{-}}} M_{s} d A_{s}^{\mu^{-}}\right) .
$$

Noting that $E_{x}^{\mu^{+}}\left(M_{0}\right)=g^{\mu}(x), e^{-A_{\zeta}^{\mu^{-}}} M_{\zeta}=e^{-A_{\zeta}^{\mu^{-}}} e^{A_{\zeta}^{\mu^{-}}}=1$ and

$$
E_{x}^{\mu^{+}}\left(\int_{0}^{\zeta} e^{-A_{s}^{\mu^{-}}} M_{s} d A_{s}^{\mu^{-}}\right)=E_{x}^{\mu^{+}}\left(\int_{0}^{\zeta} g^{\mu}\left(X_{s}\right) d A_{s}^{\mu^{-}}\right)=G^{\mu^{+}}\left(g^{\mu} \mu^{-}\right)(x)
$$

we have this lemma.

Lemma 5.4. The function $g^{\mu}$ belongs to $\mathcal{D}_{\text {loc }}(\mathcal{E}) \cap C_{b}(X)$.

Proof. On account of Lemma 5.3, we have only to prove that $G^{\mu^{+}}\left(g^{\mu} \mu^{-}\right) \in$ $\mathcal{D}_{\text {loc }}(\mathcal{E}) \cap C_{b}(X)$. First note that $g^{\mu} \mu^{-} \in \mathcal{K}_{\infty}\left(G^{\mu^{+}}\right)$. Hence, $G^{\mu^{+}}\left(g^{\mu} \mu^{-}\right) \in$ $\mathscr{B}_{b}(X)$ by [2, Proposition 2.2] and $p_{t}^{\mu+}\left(G^{\mu^{+}}\left(g^{\mu} \mu^{-}\right)\right) \in C_{b}(X)$ by the strong Feller property of $\mathbf{M}^{\mu^{+}}$. Since

$$
\begin{aligned}
\left\|G^{\mu^{+}}\left(g^{\mu} \mu^{-}\right)-p_{t}^{\mu+}\left(G^{\mu^{+}}\left(g^{\mu} \mu^{-}\right)\right)\right\|_{\infty} & =\sup _{x \in X} E_{x}^{\mu^{+}}\left(\int_{0}^{t} g^{\mu}\left(X_{s}\right) d A_{s}^{\mu^{-}}\right) \\
& \leq\left\|g^{\mu}\right\|_{\infty}\left(\sup _{x \in X} E_{x}^{\mu^{+}}\left(A_{t}^{\mu^{-}}\right)\right) \downarrow 0, \quad t \downarrow 0
\end{aligned}
$$

by $\mu^{-} \in \mathcal{K}\left(G^{\mu^{+}}\right)$and $(2.2), G^{\mu^{+}}\left(g^{\mu} \mu^{-}\right)$also belongs to $C_{b}(X)$. By the same argument as in $\left[9\right.$, Theorem 3], we can prove $G^{\mu^{+}}\left(g^{\mu} \mu^{-}\right) \in \mathcal{D}_{\text {loc }}(\mathcal{E})$.

LEmma 5.5. The Dirichlet form $\left(\mathcal{E}^{\mu, g^{\mu}}, \mathcal{D}\left(\mathcal{E}^{\mu, g^{\mu}}\right)\right)$ on $L^{2}\left(X ;\left(g^{\mu}\right)^{2} m\right)$ is transient.

Proof. Since for $u \in \mathcal{D}_{e}\left(\mathcal{E}^{\mu^{+}}\right)$

$$
\mathcal{E}^{\mu^{+}}(u, u) \geq \lambda(\mu) \int_{X} u^{2} d \mu^{-}, \quad \lambda(\mu)>1
$$

we have

$$
\mathcal{E}^{\mu}(u, u)=\mathcal{E}^{\mu^{+}}(u, u)-\int_{X} u^{2} d \mu^{-} \geq\left(\frac{\lambda(\mu)-1}{\lambda(\mu)}\right) \cdot \mathcal{E}^{\mu^{+}}(u, u) .
$$

Since $\left(\mathcal{E}^{\mu^{+}}, \mathcal{D}\left(\mathcal{E}^{\mu^{+}}\right)\right)$is transient, there exists a bounded function $g^{+}>0$ such that

$$
\int_{X} u^{2} g^{+} d m \leq \mathcal{E}^{\mu^{+}}(u, u), \quad u \in \mathcal{D}_{e}\left(\mathcal{E}^{\mu^{+}}\right)
$$


and thus for $v \in \mathcal{D}_{e}\left(\mathcal{E}^{\mu, g^{\mu}}\right)$

$$
\begin{aligned}
\int_{X} v^{2} g^{+}\left(g^{\mu}\right)^{2} d m & =\int_{X}\left(g^{\mu} v\right)^{2} g^{+} d m \leq \mathcal{E}^{\mu^{+}}\left(g^{\mu} v, g^{\mu} v\right) \\
& \leq\left(\frac{\lambda(\mu)}{\lambda(\mu)-1}\right) \cdot \mathcal{E}^{\mu}\left(g^{\mu} v, g^{\mu} v\right)=\left(\frac{\lambda(\mu)}{\lambda(\mu)-1}\right) \cdot \mathcal{E}^{\mu, g^{\mu}}(v, v)
\end{aligned}
$$

which leads us to this lemma.

Note that if $\mu^{+}$is non-trivial, $\mu^{+} \not \equiv 0$, then $\mathbf{M}^{\mu, g^{\mu}}$ is not conservative, $p_{t}^{\mu, g^{\mu}} 1(x)<1$. Indeed, we show in the proof of Lemma 5.2 that

$$
p_{t}^{\mu} g^{\mu}(x)=E_{x}^{\mu^{+}}\left(e^{A_{\zeta}^{\mu^{-}}} ; t<\zeta\right) .
$$

Since $P_{x}^{\mu^{+}}(\zeta=\infty)<1$ for all $x \in X$,

$$
\lim _{t \rightarrow \infty} p_{t}^{\mu} g^{\mu}(x)=E_{x}^{\mu^{+}}\left(e^{A_{\zeta}^{\mu^{-}}} ; \zeta=\infty\right)<g^{\mu}(x),
$$

and thus $p_{t}^{\mu, g^{\mu}} 1(x)<1$. As a result, the Dirichlet form $\left(\mathcal{E}^{\mu, g^{\mu}}, \mathcal{D}\left(\mathcal{E}^{\mu, g^{\mu}}\right)\right)$ is transient.

Lemma 5.5 leads us to the next theorem.

THEOREM 5.6. If $\lambda(\mu)>1$, the Schrödinger form $\left(\mathcal{E}^{\mu}, \mathcal{D}\left(\mathcal{E}^{\mu}\right)\right)$ is subcritical.

Next two lemmas are used to show the existence of a bounded function in $\mathcal{H}^{+}(\mu)$ when $\left(\mathcal{E}^{\mu}, \mathcal{D}\left(\mathcal{E}^{\mu}\right)\right)$ is critical.

LEMMA 5.7. Let $\mu=\mu^{+}-\mu^{-} \in \mathcal{K}_{\text {loc }}-\mathcal{K}_{\infty}\left(G^{\mu^{+}}\right)$. If $\lambda(\mu)>1$, then for any compact set $K$,

$$
\sup _{x \in K} G^{\mu} 1_{K}(x)<\infty
$$

Proof. Let $g^{\mu}$ be the gauge function in (5.3). The Markov semigroup $p_{t}^{\mu, g^{\mu}}$ is strong Feller. Indeed, for $f \in \mathscr{B}_{b}(X), p_{t}^{\mu}\left(g^{\mu} f\right)$ belongs to $C_{b}(X)$ by the strong Feller property of $p_{t}^{\mu}$. Hence $p_{t}^{\mu, g^{\mu}} f \in \mathscr{B}_{b}(X) \cap C(X)=C_{b}(X)$. As a result, $p_{t}^{\mu, g^{\mu}}$ is a transient semigroup with (LSC). We now see from [7, Corollary 2.3] that for any compact set $K, G^{\mu, g^{\mu}} 1_{K}$ is bounded, $\left\|G^{\mu, g^{\mu}} 1_{K}\right\|_{\infty}<\infty$. Noting

$$
\begin{aligned}
\inf _{x \in K} g^{\mu}(x) \cdot G^{\mu} 1_{K}(x) & \leq G^{\mu}\left(1_{K} g^{\mu}\right)(x)=g^{\mu}(x) \cdot G^{\mu, g^{\mu}} 1_{K}(x) \\
& \leq\left\|G^{\mu, g^{\mu}} 1_{K}\right\|_{\infty} \cdot g^{\mu}(x)
\end{aligned}
$$

we have by Lemma 5.4

$$
\sup _{x \in K} G^{\mu} 1_{K}(x) \leq\left\|G^{\mu, g^{\mu}} 1_{K}\right\|_{\infty} \cdot \frac{\sup _{x \in K} g^{\mu}(x)}{\inf _{x \in K} g^{\mu}(x)}<\infty .
$$


LEMMA 5.8. Let $\mu=\mu^{+}-\mu^{-} \in \mathcal{K}_{\text {loc }}-\mathcal{K}_{\infty}\left(G^{\mu^{+}}\right)$with $\lambda(\mu)>1$. Let $\nu$ be a smooth positive Radon measure and $K$ the fine support of $\nu, K=\{x \in$ $\left.X: P_{x}\left(\tau^{\nu}=0\right)=1\right\}\left(\tau^{\nu}=\inf \left\{t>0: A_{t}^{\nu}>0\right\}\right)$. Then

$$
\sup _{x \in X} G^{\mu} \nu(x) \leq \sup _{x \in K} G^{\mu} \nu(x) \cdot \sup _{x \in X} E_{x}^{\mu^{+}}\left(e^{\left.A_{\tau_{D}}^{\mu-} ; \tau_{D}<\zeta\right),}\right.
$$

where $D$ is the complement of $K$ and $\tau_{D}$ is the first exit time from $D$.

Proof. Noting that $d A_{t}^{\nu}=0$ on $\left\{t<\tau_{D}\right\}$, we have

$$
G^{\mu} \nu(x)=E_{x}\left(\int_{0}^{\zeta} e^{-A_{t}^{\mu}} d A_{t}^{\nu}\right)=E_{x}\left(\int_{\tau_{D}}^{\zeta} e^{-A_{t}^{\mu}} d A_{t}^{\nu} ; \tau_{D}<\zeta\right) .
$$

Because $\zeta=\tau_{D}+\zeta\left(\theta_{\tau_{D}}\right)$ on $\left\{\tau_{D}<\zeta\right\}, A_{\tau_{D}+t}^{\mu}=A_{\tau_{D}}^{\mu}+A_{t}^{\mu}\left(\theta_{\tau_{D}}\right)$ and $A_{\tau_{D}+t}^{\nu}=$ $A_{\tau_{D}}^{\nu}+A_{t}^{\nu}\left(\theta_{\tau_{D}}\right)$, the right-hand side of (5.6) equals

$$
\begin{aligned}
& E_{x}\left(\int_{0}^{\zeta\left(\theta_{\tau_{D}}\right)} e^{-A_{\tau_{D}+t}^{\mu}} d A_{\tau_{D}+t}^{\nu} ; \tau_{D}<\zeta\right) \\
& \quad=E_{x}\left(\int_{0}^{\zeta\left(\theta_{\tau_{D}}\right)} e^{-A_{\tau_{D}}^{\mu}-A_{t}^{\mu}\left(\theta_{\tau_{D}}\right)} d A_{t}^{\nu}\left(\theta_{\tau_{D}}\right) ; \tau_{D}<\zeta\right) \\
& \quad=E_{x}\left(e^{-A_{\tau_{D}}^{\mu} E_{X_{\tau_{D}}}}\left(\int_{0}^{\zeta} e^{-A_{t}^{\mu}} d A_{t}^{\nu}\right) ; \tau_{D}<\zeta\right)
\end{aligned}
$$

by the strong Markov property. Therefore, the right-hand side is less than or equal to

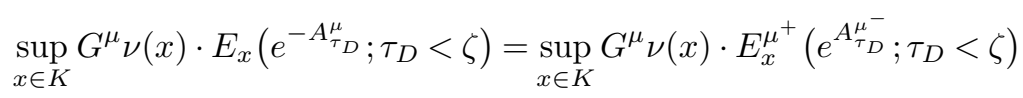

because $\tau_{D}$ equals the first hitting time of $K$ on $\left\{\tau_{D}<\zeta\right\}$.

Lemma 5.8 tells us that if $\lambda(\mu)>1$

$$
\sup _{x \in X} G^{\mu} \nu(x) \leq \sup _{x \in K} G^{\mu} \nu(x) \cdot \sup _{x \in X} g^{\mu}(x) \leq C \sup _{x \in K} G^{\mu} \nu(x)
$$

because

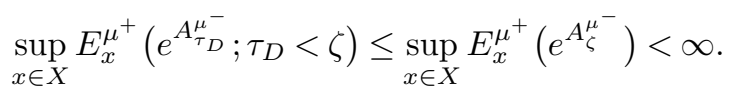

If $\mu^{-}$equals zero, $\mu=\mu^{+}$, then

$$
\sup _{x \in X} G^{\mu^{+}} \nu(x) \leq \sup _{x \in K} G^{\mu^{+}} \nu(x) \cdot \sup _{x \in X} P_{x}^{\mu^{+}}\left(\tau_{D}<\zeta\right) \leq \sup _{x \in K} G^{\mu^{+}} \nu(x) .
$$

Consequently, $\sup _{x \in X} G^{\mu^{+}} \nu(x)=\sup _{x \in K} G^{\mu^{+}} \nu(x)$, which is known as the maximum principle (e.g., [10, Theorem 1.10]). Hence, the inequality (5.5) is regarded as a version of the maximum principle for Green functions of Schrödinger operators. 
5.2. Critical case. In this subsection, we treat the case that $\lambda(\mu)=1$.

Lemma 5.9. Let $\nu \in \mathcal{K}_{\text {loc. }}$ Then $G^{\nu} \varphi$ belongs to $\mathcal{D}_{e}\left(\mathcal{E}^{\nu}\right)$ for $\varphi \in \mathscr{B}_{b, 0}^{+}$, and

$$
\mathcal{E}^{\nu}\left(G^{\nu} \varphi, f\right)=\int_{X} \varphi f d m, \quad f \in \mathcal{D}(\mathcal{E}) \cap C_{0}(X) .
$$

Proof. The function $G_{\beta}^{\nu} \varphi, \beta>0$, belongs to $\mathcal{D}\left(\mathcal{E}^{\nu}\right)$ and $G_{\beta}^{\nu} \varphi \uparrow G^{\nu} \varphi$ as $\beta \downarrow 0$. Moreover,

$$
\mathcal{E}^{\nu}\left(G_{\beta}^{\nu} \varphi, G_{\beta}^{\nu} \varphi\right) \leq \mathcal{E}_{\beta}^{\nu}\left(G_{\beta}^{\nu} \varphi, G_{\beta}^{\nu} \varphi\right)=\left(\varphi, G_{\beta}^{\nu} \varphi\right) \leq\left(\varphi, G^{\nu} \varphi\right)<\infty
$$

by Lemma 5.7. Hence, $G^{\nu} \varphi$ belongs to $\mathcal{D}_{e}\left(\mathcal{E}^{\nu}\right)$ from Lemma 2.9. Using Lemma 5.7 again, we have

$$
\mathcal{E}^{\nu}\left(G^{\nu} \varphi, f\right)=\lim _{\beta \downarrow 0} \mathcal{E}^{\nu}\left(G_{\beta}^{\nu} \varphi, f\right)=(\varphi, f)_{m}-\lim _{\beta \downarrow 0} \beta\left(G_{\beta}^{\nu} \varphi, f\right)_{m}=(\varphi, f)_{m} .
$$

We see from [14, Theorem 2.1] that the minimizer $v$ in (5.1) exists:

$$
\lambda(\mu)=\mathcal{E}(v, v)+\int_{X} v^{2} d \mu^{+}, \quad \int_{X} v^{2} d \mu^{-}=1 .
$$

We now show that a version of $v$ belongs to $\mathcal{H}^{+}$by the arguments in $[15$, Section 4]. The function $v$ is the first eigenfunction corresponding to the generator of the time changed process of $\mathbf{M}^{\mu^{+}}$by $A_{t}^{\mu^{-}}$. We see that the time changed process is irreducible and thus $v>0 \mu^{-}$-a.e. by [4, Theorem 7.3].

LEMma 5.10. The measure $v \cdot \mu^{-}$is of finite energy integral with respect to $\mathcal{E}^{\mu^{+}}$.

Proof. Let $f \in \mathcal{D}_{e}\left(\mathcal{E}^{\mu^{+}}\right)$. Then

$$
\int_{X} f v d \mu^{-} \leq\left(\int_{X} v^{2} d \mu^{-}\right)^{1 / 2}\left(\int_{X} f^{2} d \mu^{-}\right)^{1 / 2}
$$

and the right-hand side is dominated by $C \cdot \mathcal{E}^{\mu^{+}}(f, f)^{1 / 2}$ by $(2.3)$.

The function $v$ is also characterized by

$$
\mathcal{E}(v, f)+\int_{X} v f d \mu^{+}=\int_{X} v f d \mu^{-}, \quad f \in \mathcal{D}_{e}\left(\mathcal{E}^{\mu^{+}}\right) .
$$

Hence, we see from Lemma 5.10 that

$$
\mathcal{E}^{\mu^{+}}(v, f)=\int_{X} v f d \mu^{-}=\mathcal{E}^{\mu^{+}}\left(G^{\mu^{+}}\left(v \mu^{-}\right), f\right),
$$

and thus

$$
v(x)=G^{\mu^{+}}\left(v \mu^{-}\right)(x)=E_{x}\left(\int_{0}^{\zeta} \exp \left(-A_{t}^{\mu^{+}}\right) v\left(X_{t}\right) d A_{t}^{\mu^{-}}\right), \quad m \text {-a.e. }
$$


We now define

$$
h(x):=E_{x}\left(\int_{0}^{\zeta} \exp \left(-A_{t}^{\mu^{+}}\right) v\left(X_{t}\right) d A_{t}^{\mu^{-}}\right) .
$$

LEMMA 5.11. The function $h$ is finely continuous.

Proof. By the Markov property,

$$
\begin{aligned}
h\left(X_{s}\right)= & E_{X_{s}}\left(\int_{0}^{\zeta} \exp \left(-A_{t}^{\mu^{+}}\right) v\left(X_{t}\right) d A_{t}^{\mu^{-}}\right) \\
= & E_{x}\left(\int_{0}^{\zeta} \exp \left(-A_{t}^{\mu^{+}}\left(\theta_{s}\right)\right) v\left(X_{t+s}\right) d A_{t}^{\mu^{-}}\left(\theta_{s}\right) \mid \mathscr{F}_{s}\right) \\
= & \exp \left(A_{s}^{\mu^{+}}\right) E_{x}\left(\int_{0}^{\zeta} \exp \left(-A_{t}^{\mu^{+}}\right) v\left(X_{t}\right) d A_{t}^{\mu^{-}} \mid \mathscr{F}_{s}\right) \\
& -\exp \left(A_{s}^{\mu^{+}}\right) \int_{0}^{s} \exp \left(-A_{t}^{\mu^{+}}\right) v\left(X_{t}\right) d A_{t}^{\mu^{-}} .
\end{aligned}
$$

Since the first term of the right-hand side is right continuous because of the right continuity of $\mathscr{F}_{s}, h$ is finely continuous by [6, Theorem A.2.7].

Noting that $h=v$ q.e. by (5.10) and [6, Lemma 4.1.5], we have the next lemma from [6, Theorem 4.1.2].

LEMMA 5.12. The function $h$ is strictly positive and satisfies

$$
h(x)=E_{x}\left(\int_{0}^{\zeta} \exp \left(-A_{t}^{\mu^{+}}\right) h\left(X_{t}\right) d A_{t}^{\mu^{-}}\right)
$$

for all $x \in X$.

LEMmA 5.13. For $w \in \mathscr{B}_{b, 0}^{+}$with $\int_{X} w d m>0$, let $\nu=\mu+w \cdot m$. Then $\lambda(\nu)>1$.

Proof. On account of the argument above, we have a function $h^{\prime} \in \mathcal{D}_{e}\left(\mathcal{E}^{\mu^{+}}\right)$ attaining the infimum:

$$
\lambda(\nu)=\inf \left\{\mathcal{E}(u, u)+\int_{X} u^{2}\left(d \mu^{+}+w \cdot d m\right): u \in \mathcal{D}\left(\mathcal{E}^{\mu}\right), \int_{X} u^{2} d \mu^{-}=1\right\} .
$$

We can assume that $h^{\prime}$ is strictly positive. Hence, if $\lambda(\nu)=1$, then $\lambda(\mu)<1$.

LEMmA 5.14. For $w \in \mathscr{B}_{b, 0}^{+}$with $\int_{X} w d m>0$, let $\nu=\mu+w \cdot m$. The function $h$ defined in (5.11) satisfies

$$
h(x)=G^{\nu}(h w)(x) \quad \text { for any } x \in X .
$$


Proof. Noting that $\mathcal{E}^{\mu}\left(h, G_{\beta}^{\nu} \varphi\right)=0$ by $(5.9)$, we have for any $\varphi \in \mathscr{B}_{b, 0}^{+}$and any $\beta>0$

$$
\mathcal{E}^{\nu}\left(h, G_{\beta}^{\nu} \varphi\right)=\mathcal{E}^{\mu}\left(h, G_{\beta}^{\nu} \varphi\right)+\int_{X} h G_{\beta}^{\nu} \varphi \cdot w d m=\int_{X} h w G_{\beta}^{\nu} \varphi d m .
$$

On account of Lemma 5.9, we obtain, by letting $\beta \downarrow 0$

$$
\int_{X} h \varphi d m=\int_{X} h w G^{\nu} \varphi d m=\int_{X} G^{\nu}(h w) \varphi d m
$$

and thus

$$
h=G^{\nu}(h w) \quad m \text {-a.e. }
$$

In the equality above we can replace " $m$-a.e. $x$ " by "any $x$ " by the same argument as in Lemma 5.11.

LEMMA 5.15. The function $h$ is bounded.

Proof. Since $h$ is quasi-continuous, there exists a compact set $K$ with $m(K)>0$ on which $h$ is continuous. Let $\nu=\mu+1_{K} m$. Then $\lambda(\nu)>1$ and $h=G^{\nu}\left(1_{K} h\right)$ by Lemma 5.13 and Lemma 5.14. Hence, Lemma 5.8 tells us that

$$
\begin{aligned}
h(x) & =G^{\nu}\left(1_{K} h\right)(x) \leq \sup _{x \in K} G^{\nu}\left(1_{K} h\right)(x) \cdot \sup _{x \in X} E_{x}^{\nu^{+}}\left(e^{A_{\zeta}^{\nu^{-}}}\right) \\
& =\sup _{x \in K} h(x) \cdot \sup _{x \in X} g^{\nu}(x),
\end{aligned}
$$

where $\nu^{+}=\mu^{+}+1_{K} m$ and $\nu^{-}=\mu^{-}$. The right-hand side is finite because $\lambda(\nu)>1$.

LEMma 5.16. The function $h$ satisfies $p_{t}^{\mu} h=h$.

Proof. Note that there exists a generalized compact nest $\left\{F_{k}\right\}$ associated with $\mu$ because $\mu$ is a smooth Radon measure [6, p. 83]. Thus the space $\mathcal{D}_{b}\left(\mathcal{E}^{F_{n}}\right)=\left\{u \in \mathcal{D}_{b}(\mathcal{E}): u=0\right.$ q.e. on $\left.X \backslash F_{n}\right\}$ is contained in $\mathcal{D}\left(\mathcal{E}^{\mu}\right)$. Hence, it holds that $\mathcal{E}^{\mu}(h, \varphi)=0$, that is,

$$
\mathcal{E}(h, \varphi)=-\int_{X} \varphi(h \cdot d \mu) \quad \text { for any } \varphi \in \bigcup_{n=1}^{\infty} \mathcal{D}_{b}\left(\mathcal{E}^{F_{n}}\right) .
$$

We then see from [6, Theorem 5.4.2] that

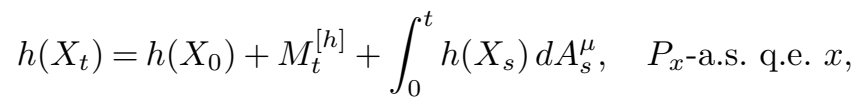

where $M_{t}^{[h]}$ is the martingale part of the Fukushima decomposition [6, Theorem 5.2.2]. Hence, we see from Itô's formula

$$
e^{-A_{t}^{\mu}} h\left(X_{t}\right)=h\left(X_{0}\right)+\int_{0}^{t} e^{-A_{s}^{\mu}} d M_{s}^{[h]}, \quad P_{x} \text {-a.s. q.e. } x \text {. }
$$


Let $\tau_{n}=\inf \left\{t>0 ; A_{t}^{\mu^{+}}>n\right\} \quad(n=1,2, \ldots)$. Then $\int_{0}^{\tau_{n} \wedge t} e^{-A_{s}^{\mu}} d M_{s}^{[h]}$ is a martingale, and

$$
h(x)=E_{x}\left(e^{-A_{\tau_{n} \wedge t}^{\mu}} h\left(X_{\tau_{n} \wedge t}\right)\right)=E_{x}^{\mu^{+}}\left(e^{A_{\tau_{n} \wedge t}^{\mu^{-}}} h\left(X_{\tau_{n} \wedge t}\right)\right) .
$$

Note that

$$
e^{A_{\tau_{n} \wedge t}^{\mu^{-}}} h\left(X_{\tau_{n} \wedge t}\right) \leq\|h\|_{\infty} \cdot e^{A_{\zeta}^{\mu^{-}}} \in L^{1}\left(P_{x}^{\mu^{+}}\right)
$$

and that $\tau_{n} \rightarrow \infty$ as $n \rightarrow \infty P_{x}^{\mu^{+}}$-a.s. We then see that by the dominated convergence theorem

$$
h(x)=\lim _{n \rightarrow \infty} E_{x}^{\mu^{+}}\left(e^{A_{\tau_{n} \wedge t}^{\mu^{-}}} h\left(X_{\tau_{n} \wedge t}\right)\right)=E_{x}^{\mu^{+}}\left(e^{A_{t}^{\mu^{-}}} h\left(X_{t}\right)\right)=p_{t}^{\mu} h(x), \quad \text { q.e. } x,
$$

and thus for all $x$.

The next corollary follows from Lemma 5.16 and the strong Feller property of $p_{t}^{\mu}$.

Corollary 5.17. The function $h$ is in $C_{b}(X)$.

We see from Corollary 5.17 that the function $h$ is an element of $\mathcal{D}_{\text {loc }}(\mathcal{E})$ because a bounded function $f$ in $\mathcal{D}_{e}(\mathcal{E})$ belongs to $\mathcal{D}_{\text {loc }}(\mathcal{E})$. Indeed, let $\left\{f_{n}\right\}$ be an approximating function of $f$, that is, $f_{n}(x) \rightarrow f(x) m$-a.e. and $\sup _{n} \mathcal{E}\left(f_{n}, f_{n}\right)<\infty$. We may assume $\left|f_{n}(x)\right| \leq\|f\|_{\infty}$. Let $D$ and $G$ be relatively compact open sets such that $G \subset \bar{G} \subset D$ and $\varphi$ a function in $\mathcal{D}(\mathcal{E}) \cap C_{0}(D)$ such that $\varphi=1$ on $G$. Then $f_{n} \varphi \rightarrow f \varphi m$-a.e. and

$$
\sup _{n} \mathcal{E}\left(f_{n} \varphi, f_{n} \varphi\right)^{1 / 2} \leq\|f\|_{\infty} \cdot \mathcal{E}(\varphi, \varphi)^{1 / 2}+\|\varphi\|_{\infty} \cdot \sup _{n} \mathcal{E}\left(f_{n}, f_{n}\right)^{1 / 2}<\infty
$$

Hence, $f \varphi$ belongs to $\mathcal{D}_{e}(\mathcal{E}) \cap L^{2}(X ; m)$ and so to $\mathcal{D}(\mathcal{E})$ by $[6$, Theorem 1.5.2(iii)]. Since $f=f \varphi$ on $G, f$ belongs to $\mathcal{D}_{\text {loc }}(\mathcal{E})$.

The function $h$ belongs to $\mathcal{D}_{e}\left(\mathcal{E}^{\mu^{+}}\right)\left(\subset \mathcal{D}_{e}(\mathcal{E})\right)$ and $\mathcal{E}^{\mu}(h, h)=0$. Consequently, the constant function 1 belongs to $\mathcal{D}_{e}\left(\mathcal{E}^{\mu, h}\right)$ and $\mathcal{E}^{\mu, h}(1,1)=0$; this implies that $\left(\mathcal{E}^{\mu, h}, \mathcal{D}\left(\mathcal{E}^{\mu, h}\right)\right)$ is recurrent. Therefore, we have the next theorem.

THEOREM 5.18. If $\lambda(\mu)=1$, then the Schrödinger form $\left(\mathcal{E}^{\mu}, \mathcal{D}\left(\mathcal{E}^{\mu}\right)\right)$ is critical.

We can show that $\lambda(\mu)<1$ if and only if $\inf \left\{\mathcal{E}^{\mu}(u, u) ; \int_{X} u^{2} d m=1\right\}<0$ [15, Lemma 2.2]. Namely, if $\lambda(\mu)<1$, then $\mathcal{E}^{\mu}$ is not non-negative definite. Hence the Schrödinger form $\left(\mathcal{E}^{\mu}, \mathcal{D}\left(\mathcal{E}^{\mu}\right)\right)$ is supercritical by Remark 4.5. Therefore, we can summarize these facts above as follows.

ThEOREM 5.19. Let $\mu \in \mathcal{K}_{\text {loc }}-\mathcal{K}_{\infty}\left(G^{\mu^{+}}\right)$. Then

(i) $\left(\mathcal{E}^{\mu}, \mathcal{D}\left(\mathcal{E}^{\mu}\right)\right)$ is subcritical if and only if $\lambda(\mu)>1$;

(ii) $\left(\mathcal{E}^{\mu}, \mathcal{D}\left(\mathcal{E}^{\mu}\right)\right)$ is critical if and only if $\lambda(\mu)=1$;

(iii) $\left(\mathcal{E}^{\mu}, \mathcal{D}\left(\mathcal{E}^{\mu}\right)\right)$ is supercritical if and only if $\lambda(\mu)<1$. 
Let us consider the symmetric $\alpha$-stable process $\mathbf{M}^{\alpha}$ on $\mathbb{R}^{d}$ with $0<\alpha<2$. We assume that $\alpha<d$, that is, $\mathbf{M}^{\alpha}$ is transient. Let $\left(\mathcal{E}^{(\alpha)}, \mathcal{D}\left(\mathcal{E}^{(\alpha)}\right)\right)$ be the Dirichlet form generated by $\mathbf{M}^{\alpha}$ :

$$
\left\{\begin{array}{l}
\mathcal{E}^{(\alpha)}(u, v)=\frac{1}{2} \mathcal{A}(d, \alpha) \iint_{\mathbb{R}^{d} \times \mathbb{R}^{d} \backslash \triangle} \frac{(u(x)-u(y))(v(x)-v(y))}{|x-y|^{d+\alpha}} d x d y, \\
\mathcal{D}\left(\mathcal{E}^{(\alpha)}\right)=\left\{u \in L^{2}\left(\mathbb{R}^{d}\right): \iint_{\mathbb{R}^{d} \times \mathbb{R}^{d} \backslash \triangle} \frac{(u(x)-u(y))^{2}}{|x-y|^{d+\alpha}} d x d y<\infty\right\},
\end{array}\right.
$$

where $\triangle=\left\{(x, x): x \in \mathbb{R}^{d}\right\}$ and

$$
\mathcal{A}(d, \alpha)=\frac{\alpha 2^{d-1} \Gamma\left(\frac{\alpha+d}{2}\right)}{\pi^{d / 2} \Gamma\left(1-\frac{\alpha}{2}\right)}
$$

[6, Example 1.4.1]. Let $\mu$ be a measure in the Green-tight class of $\mathbf{M}^{\alpha}$. Suppose that the Schrödinger form $\mathcal{E}^{(\alpha)}(u, u)-\int_{\mathbb{R}^{d}} u^{2} d \mu$ is critical. Then

$$
\int_{\mathbb{R}^{d}} u^{2} d \mu \leq \mathcal{E}^{(\alpha)}(u, u) \quad \text { for any } u \in \mathcal{D}_{e}(\mathcal{E})
$$

and the two sides are equal if and only if $u$ equals the function $h$ constructed in Section 5.2. On account of Theorem 3.2, we can refine the inequality above as follows:

$$
\int_{\mathbb{R}^{d}} u^{2} d \mu+\int_{\mathbb{R}^{d}}\left(u-D_{h}(u) h\right)^{2} g d x \leq \mathcal{E}^{(\alpha)}(u, u) \quad \text { for any } u \in \mathcal{D}_{e}(\mathcal{E}) .
$$

Note that $D_{h}(h)=1$ by the definition of $D_{h}(u)$.

\section{REFERENCES}

[1] S. Albeverio, P. Blanchard and Z.-M. Ma, Feynman-Kac semigroups in terms of signed smooth measures, Random partial differential equations (Oberwolfach, 1989), Birkhäuser, Basel, 1991, pp. 1-31. MR 1185735

[2] Z.-Q. Chen, Gaugeability and conditional gaugeability, Trans. Amer. Math. Soc. 354 (2002), 4639-4679. MR 1926893

[3] Z.-Q. Chen and K. Kuwae, On doubly Feller property, Osaka J. Math. 46 (2009), 909-930. MR 2604914

[4] E. B. Davies, One parameter semigroup, London Mathematical Society Monographs, Academic Press, New York, 1980. MR 0591851

[5] R. M. Dudley, Real analysis and probability, Cambridge Studies in Advanced Mathematics, vol. 74, Cambridge University Press, Cambridge, 2002. MR 1932358

[6] M. Fukushima, Y. Oshima and M. Takeda, Dirichlet forms and symmetric Markov processes, 2nd ed., Walter de Gruyter, Berlin, 2011. MR 2778606

[7] R. K. Getoor, Transience and recurrence of Markov processes, Seminar on Probability, XIV (Paris, 1978/1979), Lecture Notes in Math., vol. 784, Springer, Berlin, 1980, pp. 397-409. MR 0580144

[8] D. Kim and K. Kuwae, Analytic characterizations of gaugeability for generalized Feynman-Kac functionals, preprint.

[9] D. Kim and K. Kuwae, On a stability of heat kernel estimates under generalized non-local Feynman-Kac perturbations for stable-like processes, Festschrift Masatoshi Fukushima: In honor of Masatoshi Fukushima's Sanju, Interdisciplinary Mathematical Sciences, vol. 17, World Sci. Publ., Hackensack, NJ, 2014, pp. 287-305. 
[10] N. S. Landkof, Foundations of modern potential theory, Springer-Verlag, New York, 1972. MR 0350027

[11] Y. Pinchover and K. Tintarev, A ground state alternative for singular Schrödinger operators, J. Funct. Anal. 230 (2006), 65-77. MR 2184184

[12] M. L. Silverstein, Symmetric Markov processes, Lecture Notes in Math., vol. 426, Springer-Verlag, Berlin, 1974. MR 0386032

[13] P. Stollmann and J. Voigt, Perturbation of Dirichlet forms by measures, Potential Anal. 5 (1996), 109-138. MR 1378151

[14] M. Takeda, A variational formula for Dirichlet forms and existence of ground states, J. Funct. Anal. 266 (2014), 660-675. MR 3132724

[15] M. Takeda and K. Tsuchida, Differentiability of spectral functions for symmetric $\alpha$ stable processes, Trans. Amer. Math. Soc. 359 (2007), 4031-4054. MR 2302522

Masayoshi Takeda, Mathematical Institute, Tohoku University, Aoba, Sendai, 980-8578, JAPAN

E-mail address: takeda@math.tohoku.ac.jp 